YITP-13-14

IPMU13-0045

\title{
Holographic Local Quenches and Entanglement Density
}

\author{
Masahiro Nozaki ${ }^{a}$, Tokiro Numasawa ${ }^{a}$, and Tadashi Takayanagi ${ }^{a, b}$ \\ ${ }^{a}$ Yukawa Institute for Theoretical Physics, Kyoto University, \\ Kitashirakawa Oiwakecho, Sakyo-ku, Kyoto 606-8502, Japan \\ ${ }^{b}$ Kavli Institute for the Physics and Mathematics of the Universe, \\ University of Tokyo, Kashiwa, Chiba 277-8582, Japan
}

\begin{abstract}
We propose a free falling particle in an AdS space as a holographic model of local quench. Local quenches are triggered by local excitations in a given quantum system. We calculate the time-evolution of holographic entanglement entropy. We confirm a logarithmic time-evolution, which is known to be typical in two dimensional local quenches. To study the structure of quantum entanglement in general quantum systems, we introduce a new quantity which we call entanglement density and apply this analysis to quantum quenches. We show that this quantity is directly related to the energy density in a small size limit. Moreover, we find a simple relationship between the amount of quantum information possessed by a massive object and its total energy based on the AdS/CFT.
\end{abstract}




\section{Contents}

1 Introduction

2 Holographic Local Quenches as Falling Particles 3

2.1 A Falling Massive Particle in AdS . . . . . . . . . . . . . . . . . . . . . . . . . . . .

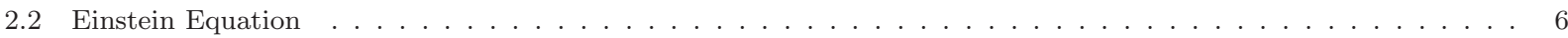

2.3 Coordinate Transformation from Global AdS to Poincare AdS . . . . . . . . . . . . . . . . . . . 6

2.4 Back-reacted Metric for a Falling Massive Particle . . . . . . . . . . . . . . . . . . . . . 7

2.5 Holographic Energy Stress Tensor . . . . . . . . . . . . . . . . . . . . . . . . . . . . .

3 Perturbative Analysis of Holographic Entanglement Entropy under Local Quenches 9

3.1 Perturbative Calculations of HEE under Local Quenches . . . . . . . . . . . . . . . . . . . . 11

3.2 Explicit Calculations of HEE under Local Quenches . . . . . . . . . . . . . . . . . . . . 11

3.3 Small Subsystem Limit: An Analogue of the first law of thermodynamics . . . . . . . . . . . . . . . . . 14

3.4 Large Subsystem Limit . . . . . . . . . . . . . . . . . . . . . . . . . . . . . . . . . . . . . 14

4 Exact Holographic Entanglement Entropy for 2d Local Quenches 15

4.1 Symmetric Intervals _. . . . . . . . . . . . . . . . . . . . . . . . . . . . . . . 15

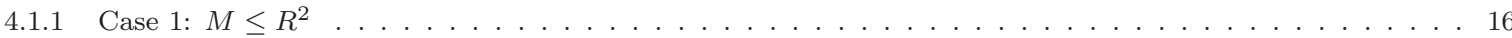

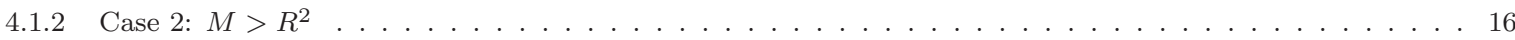

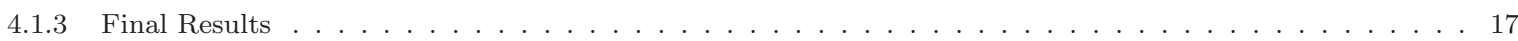

4.1 .4 Thermal Local Quenches _. . . . . . . . . . . . . . . . . . . . . . . . . . . . 18

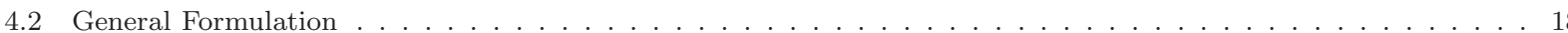

4.3 An Interval with An Excited End Point . . . . . . . . . . . . . . . . . . . . . . . 19

4.3 .1 Semi Infinite Limit $l \rightarrow \infty \ldots \ldots \ldots \ldots \ldots \ldots$

4.4 HEE for General Intervals . . . . . . . . . . . . . . . . . . . . . . . . . . . . . . 21

5 Entanglement Density 2

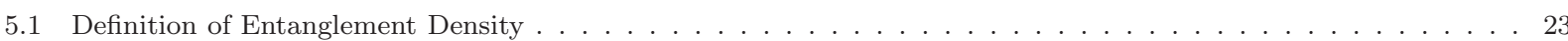

5.2 Strong Subadditivity and Positivity of Entanglement Density . . . . . . . . . . . . . . . . . . . 24

5.3 Conservation Law . . . . . . . . . . . . . . . . . . . . . . . . . . . . . 25

5.4 Entanglement Density for Global Quenches . . . . . . . . . . . . . . . . . . . . . . 26

5.5 Entanglement Density for Holographic Local Quenches . . . . . . . . . . . . . . . . . . . 26

6 Quantum Information, Thermodynamics and Gravity 2

6.1 Total Amount of Quantum Information . . . . . . . . . . . . . . . . . . . . . . . . 29

6.2 Entanglement Renormalization and Origin of Gravitational Force . . . . . . . . . . . . . . . . . . 32

7 Conclusions and Discussions

A Perturbative Construction of Gravity Duals

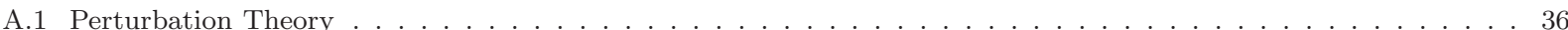

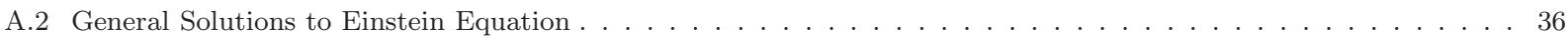

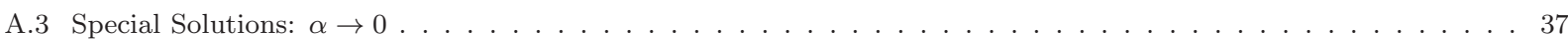




\section{Introduction}

Quantum entanglement provides us with a powerful method of investigating various quantum states and classifying their quantum structures. Moreover, quantum entanglement is a useful tool when we would like to study excited quantum systems which are far from thermal equilibrium, for example, those under thermalization processes. Even though in such systems we cannot define the thermodynamical entropy and temperature etc, we can always define the entanglement entropy (refer to e.g. the reviews [1, 2, 3, 4, 5]).

Quantum quenches provide us with ideal setups to study thermalizations of quantum systems, which can be realized even in real experiments such as cold atoms. They are unitary evolutions of pure states triggered by sudden change of parameters such as mass gaps or coupling constants [6, 7, 8]. By using the AdS/CFT [9, 10, 11], we can relate this problem to the dynamics of gravitational theories [12, 13, 14, 15].

One typical class of quantum quenches is called global quenches and they occur from homogeneous changes of parameters [6]. Holographic duals of global quenches are dual to black hole formations as have been discussed in [12, 14]. A useful quantity to understand how thermalizations occur is the entanglement entropy [6]. The holographic entanglement entropy (HEE) [16, 17, 5] has been calculated for various quantum quenches [13, 18, 19. Refer also to [20] for a computation of HEE for a stationary system which is described by an excited pure state dual to an AdS plane wave. In general, non-local probes such as the entanglement entropy are useful to measure the thermalization time. On the other hand, local quantities such as an expectation value of energy momentum tensor shows an immediate thermalization and is not suitable to see if a given system is completely thermalized [18]. In this sense, the entanglement entropy can serve as a non-equilibrium substitute of thermodynamical entropy.

Another class of quantum quenches is the local quench and this is triggered by a shift of parameters within a localized region or simply at a point. One of the aims of the present paper is to provide a simple construction of holographic dual for local quench and is to calculate the holographic entanglement entropy. A local quench shows how localized excitations in a given quantum system propagate to other spatial regions. Local quenches have been studied in two dimensional CFTs [7, 8]. However, local quenches in higher dimensions have not been understood well. This partially motivates us to study the local quenches in AdS/CFT, which often allows us higher dimensional calculations.

We will argue that a simple holographic description of a system just after the local quench is a free falling particle-like object in an AdS space. It is pulled into the horizon of AdS space due to the gravitational force and this is the reason why we observe the non-trivial time-dependence 
of entanglement entropy. Therefore this problem is deeply connected to a fundamental question: what is the non-gravitational (or CFT) counterpart of gravitational force via holography? We will suggest an intuitive answer to this question in the end.

The time evolution of quantum entanglement under local quenches is more complicated than the global quenches because it is inhomogeneous. To understand its structure clearly we introduce a new quantity which we call entanglement density. It is defined by taking the derivatives of the entanglement entropy $S_{A}$ with respect to the positions of two boundary points of the subsystem $A$. This quantity counts the number of entangled pairs at a given position. The strong subadditivity guarantees that this quantity is always positive. As we will see this analysis reveals the detailed structure of quantum entanglement under local quenches as well as global quenches.

One more motivation to study local quenches is to estimate the amount of quantum information possessed by a massive object or radiations. We will employ the entanglement entropy for local quenches in order to measure the amount of information included in a localized excited lump. We will evaluate this quantity by using the holographic entanglement entropy (HEE) and obtain the simple conclusion that it is given by the total energy of the object times its size up to a numerical factor.

The paper is organized as follows: In section two, we explain our holographic setup of local quench using a free falling particle in $\mathrm{AdS}_{d+1}$. We calculate the holographic energy stress tensors in this model. In section three, we compute the holographic entanglement entropy for $d=2,3,4$ assuming that the back-reaction due to the falling particle is very small. In section four, we perform an exact analysis of holographic entanglement entropy for $d=2$. In section 5 , we introduce a new quantity which we call entanglement density and we investigate the evolution of quantum entanglement structures under local quenches by using this. In section 6 , we study the relation between the amount of information of an object and its total energy using the results in the previous sections. We also interpret our results of local quenches using the idea of the entanglement renormalization and discuss the holographic interpretation of gravitational force. In section 7 we summarize our conclusions and discuss future problems. In appendix A, we show an explicit perturbative calculation of back-reactions due to the falling particle.

\section{Holographic Local Quenches as Falling Particles}

Local quenches in quantum systems are triggered by a sudden local change of the Hamiltonian at a specific time. One typical class of examples will be joining two separated semi-infinite systems at each endpoint as studied in [7, 8] (see the upper picture in Fig,1). When this quench process happens, an interaction between two endpoints is instantaneously introduced. From the viewpoint 


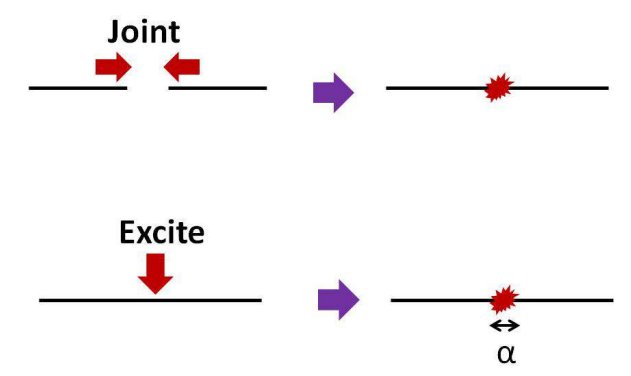

Figure 1: Setups of local quenches. The upper picture describes a process of jointing two systems which are defined on semi-infinite lines. The lower one describes localized excitations on an infinitely extended system. We define the parameter $\alpha$ which measures the size of excited region at the beginning of the local quench.

of the new Hamiltonian, an locally excited state is generated just after this local quench. Therefore we can generally characterize a local quench by local excitations. These excitations will propagate to other regions under the time-evolution.

Now we would like to construct gravity duals for local quenches via the AdS/CFT. Even though we have not found a simple gravity dual of the original model i.e. joining two CFTs, it is not difficult to find a holographic model for local excitations. It is given by a falling massive particle in a Poincare AdS space (see Fig (2). At $t=0$, the particle is situated near the AdS boundary and its back-reaction to the metric is highly localized near the particle. Under its time evolution it falls into the AdS horizon and its back-reaction spreads out. In the dual CFT, at $t=0$, the excitations are concentrated in a small localized region (see the lower picture in Fig,1), whose radius is defined to be $\alpha$. Therefore we can regard this state at $t=0$ as the one just after the local quench. Later the excitations expand at the speed of light as we will see e.g. from its holographic energy stress tensor later. In this way, we can regard this setup as a gravity dual of local quench.

We will employ the beautiful construction of back-reacted solutions found by Horowitz and Itzhaki in [21]. The basic idea is to start from a black hole in a global AdS space and map it into a Poincare AdS by the coordinate transformation. This leads to a falling black hole solution. Though we are thinking of a massive particle with a finite size or equally a star, instead of a black hole, the asymptotic solution which is outside of the star is the same as that for a black hole as usual. 

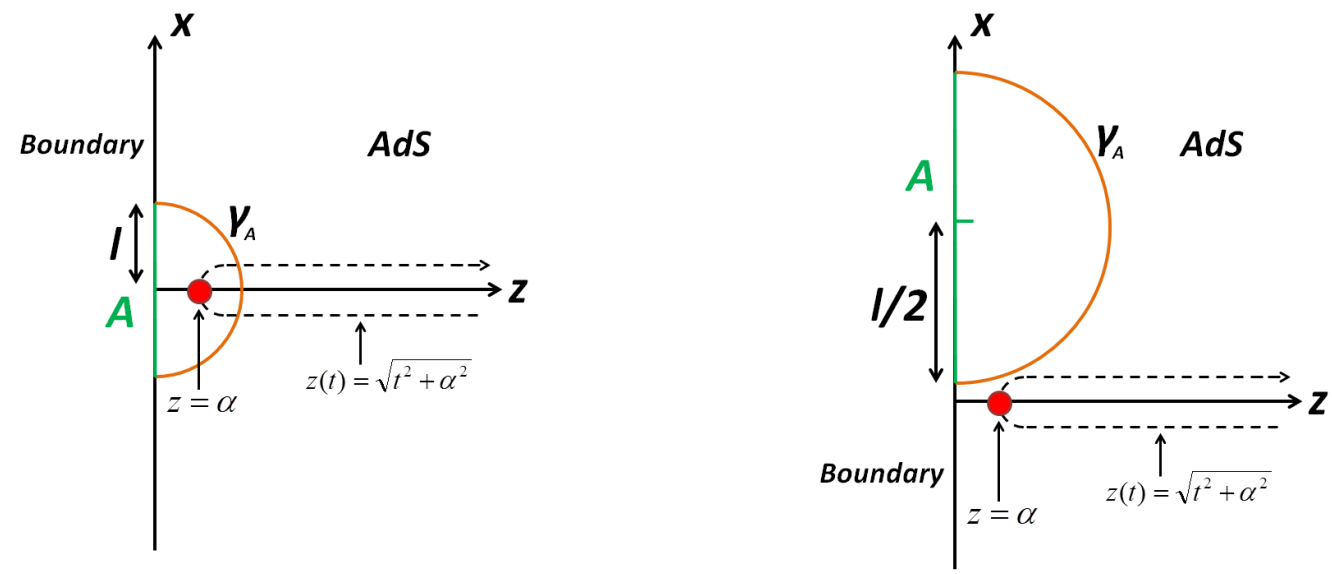

Figure 2: A falling massive particle in AdS and the calculation of holographic entanglement entropy for two different choices of the subsystem $A$. It is clear from this picture that the back reaction due to the falling particle gets significant when $l=z(t)$ in the left picture and $t=0$ in the right one because the particle is on top of $\gamma_{A}$.

\subsection{A Falling Massive Particle in AdS}

Consider a $d+1$ dimensional AdS space $\left(\mathrm{AdS}_{d+1}\right)$ in the Poincare coordinate

$$
d s^{2}=R^{2}\left(\frac{d z^{2}-d t^{2}+\sum_{i=1}^{d-1} d x_{i}^{2}}{z^{2}}\right) .
$$

The radius of AdS is defined to be $R$ and the coordinate of AdS is represented by $x^{\mu}=\left(z, t, x_{1}, \cdots\right.$ $\left.\cdot, x_{d-1}\right)$.

In this AdS space, we introduce a massive object (mass $m$ ) with a very small size which is larger than the Schwartzschild radius. Its motion in the AdS space is described by the trajectory $x^{\mu}=X^{\mu}(\tau)$. In general, the action of a particle with mass $m$ in a spacetime defined by the metric $g_{\mu \nu}$ is given by

$$
S_{p}=-m \int d \tau \int d x^{d+1} \delta^{(d+1)}\left(x^{\mu}-X^{\mu}(\tau)\right) \sqrt{-g_{\mu \nu}(x) \cdot \partial_{\tau} X^{\mu}(\tau) \cdot \partial_{\tau} X^{\nu}(\tau)} .
$$

We assume that the particle is situated at $X^{i}=0$ and we gauge fix by setting $X^{t}(\tau)=\tau$. Then the trajectory is specified by the function $X^{z}(\tau)=z(\tau)$. In the pure AdS background (2.1), the action looks like

$$
S=-m R \int d t \frac{\sqrt{1-\dot{z}(t)^{2}}}{z(t)} .
$$

The solution to the equation of motion derived from (2.3) is given by

$$
z(t)=\sqrt{\left(t-t_{0}\right)^{2}+\alpha^{2}},
$$


where $t_{0}$ and $\alpha$ are integration constants. Below we will set $t_{0}=0$ by using the time translation invariance. When $t<0$ the particle moves from the horizon to the boundary. It reaches $z=\alpha$ at $t=0$. Later $(t>0)$, it again falls into the horizon as depicted in Fig, 2 . Thus the energy of the particle in the AdS space is calculated as

$$
E=\frac{m R}{\alpha}
$$

\subsection{Einstein Equation}

The gravity action coupled to the massive particle reads

$$
S_{\text {tot }}=\frac{1}{16 \pi G_{N}} \int d x^{d+1} \sqrt{-g}(\mathcal{R}-2 \Lambda)+S_{p},
$$

where the cosmological constant is given by $\Lambda=-\frac{d(d-1)}{2 R^{2}}$ and $G_{N}$ is the Newton constant.

The equation of motion becomes

$$
\mathcal{R}^{\mu \nu}-\frac{1}{2} g^{\mu \nu} \mathcal{R}+\Lambda g^{\mu \nu}=\mathcal{T}^{\mu \nu},
$$

where the bulk energy-stress tensor $\mathcal{T}^{\mu \nu}$ is given by

$$
\mathcal{T}^{\mu \nu}=\frac{8 \pi m G_{N}}{\sqrt{-g}} \cdot \frac{\partial_{t} X^{\mu} \partial_{t} X^{\nu}}{\sqrt{-g_{\mu \nu} \cdot \partial_{t} X^{\mu}(t) \cdot \partial_{t} X^{\nu}(t)}} \cdot \delta(z-z(t)) \cdot \delta^{d-1}\left(x_{i}\right) .
$$

We will show a direct perturbative calculations of this back-reaction in appendix A. However, below we will take a different step in order to analytically construct the back-reacted solutions. See the paper [22 for analytical calculations of back-reactions to a scalar field in an AdS space. Refer also to 23] for a more extensive analysis and a relation to expanding qluon plasmas, where the back-reacted solutions are called conformal solitons (see [24] for spacetime structures of conformal solitons).

\subsection{Coordinate Transformation from Global AdS to Poincare AdS}

Now consider the global $\mathrm{AdS}_{d+1}$ space defined by the metric

$$
d s^{2}=-\left(R^{2}+r^{2}\right) d \tau^{2}+\frac{R^{2} d r^{2}}{R^{2}+r^{2}}+r^{2} d \Omega_{d-1}^{2} .
$$

We can show this is (locally) equivalent to the Poincare $A d S_{d+1}$ space (2.1) via the following coordinate transformation:

$$
\begin{aligned}
& \sqrt{R^{2}+r^{2}} \cos \tau=\frac{R^{2} e^{\beta}+e^{-\beta}\left(z^{2}+x^{2}-t^{2}\right)}{2 z}, \\
& \sqrt{R^{2}+r^{2}} \sin \tau=\frac{R t}{z} \\
& r \Omega_{i}=\frac{R x_{i}}{z} \quad(i=1,2, \cdots, d-1), \\
& r \Omega_{d}=\frac{-R^{2} e^{\beta}+e^{-\beta}\left(z^{2}+x^{2}-t^{2}\right)}{2 z} .
\end{aligned}
$$


Here, the coordinate of $S^{d-1}$ is described by $\left(\Omega_{1}, \Omega_{2}, \cdots, \Omega_{d}\right)$ such that $\sum_{i=1}^{d}\left(\Omega_{i}\right)^{2}=1$. Also we defined $x^{2}=\sum_{i=1}^{d-1} x_{i}^{2}$. The arbitrary constant $\beta$ is introduced for the later purpose, which corresponds to the boost transformation of $S O(2, d)$ symmetry. If we set $\beta=0$, (2.10) is reduced to the standard one which can be found in e.g. [11].

\subsection{Back-reacted Metric for a Falling Massive Particle}

In the global coordinate, we can consider a static particle situated at $r=0$. Following the idea in [21], we would like to map it into the Poincare AdS. After the coordinate transformation (2.10), its trajectory is mapped into

$$
x_{i}=0, \quad z^{2}-t^{2}=R^{2} e^{2 \beta} .
$$

Thus this corresponds to the previous trajectory (2.4) with the identification

$$
\alpha=R e^{\beta} .
$$

The back-reacted geometry outside of the massive object is obtained from the AdS black hole solution [25]:

$$
d s^{2}=-\left(r^{2}+R^{2}-\frac{M}{r^{d-2}}\right) d \tau^{2}+\frac{R^{2} d r^{2}}{R^{2}+r^{2}-M / r^{d-2}}+r^{2} d \Omega_{d-1}^{2} .
$$

Note that in the $\mathrm{AdS}_{3}$ case $(d=2)$, the solution (2.13) for $M<R^{2}$ is not a black hole solution but a solution with a deficit angle 1 The mass parameter $M$ in (2.13) is related to the mass $m$ of the particle via

$$
m=\frac{(d-1) \pi^{d / 2-1}}{8 \Gamma(d / 2)} \cdot \frac{M}{G_{N} R^{2}} .
$$

Therefore, we can find the back-reacted metric by performing the coordinate transformation (2.10) to the metric (2.13). This can be done in a straightforward manner by noting

$$
\begin{aligned}
& r=\frac{1}{2 z} \sqrt{R^{4} e^{2 \beta}+e^{-2 \beta}\left(z^{2}+x_{i}^{2}-t^{2}\right)^{2}-2 R^{2}\left(z^{2}-x^{2}-t^{2}\right)}, \\
& d \tau^{2}=d(\cos \tau)^{2}+d(\sin \tau)^{2}, \quad d \Omega_{d-1}^{2}=\sum_{i=1}^{d}\left(d \Omega_{i}\right)^{2} .
\end{aligned}
$$

\subsection{Holographic Energy Stress Tensor}

One way to understand the time evolution of the $\mathrm{CFT}_{d}$ state dual to the falling particle in $\mathrm{AdS}_{d+1}$, is to calculate the holographic energy stress tensor. For this purpose, it is useful to employ the Fefferman-Graham gauge of the coordinates given by the expression

$$
d s^{2}=R^{2} \cdot \frac{d z^{2}+g_{a b}(x, z) d x^{a} d x^{b}}{z^{2}} .
$$

\footnotetext{
${ }^{1}$ We do not have to worry about the singularity because we replace the region near it with a star solution.
} 
where $x^{a}=\left(t, x_{i}\right)$. We are considering the case where the boundary metric $g_{a b}(x, 0)$ coincides with the flat Minkowski metric $\eta_{a b}$ as in the Poincare AdS. Then near the AdS boundary $z=0, g_{a b}(x, z)$ behaves like

$$
g_{a b}(x, z)=\eta_{a b}+t_{a b} z^{d}+O\left(z^{d+1}\right) .
$$

In this setup, the holographic energy stress tensor [26] is calculated from the formula:

$$
T_{a b}=\frac{d \cdot R^{d-1}}{16 \pi G_{N}} \cdot t_{a b}
$$

Note that the metric we find from the coordinate transformation (2.10) of (2.13) is not in the form of the Fefferman-Graham gauge (2.16). Thus we need to perform a coordinate transformation further to achieve this gauge so that we can employ (2.18). It is also useful to define the light-cone coordinate $u=t-\rho$ and $v=t+\rho$, where $\rho=x_{1}$ for $d=2$ and $\rho=\sqrt{\sum_{i=1}^{d-1} x_{i}^{2}}$ for $d>2$. Finally we obtain the following energy stress tensor $T_{a b}$ for $d=2,3,4$ :

$$
\begin{aligned}
& d=2\left(\mathrm{AdS}_{3}\right): \\
& T_{u u}=\frac{M \alpha^{2}}{8 \pi G_{N} R\left(u^{2}+\alpha^{2}\right)^{2}}, \quad T_{v v}=\frac{M \alpha^{2}}{8 \pi G_{N} R\left(v^{2}+\alpha^{2}\right)^{2}}, \quad T_{u v}=0 . \\
& d=3\left(\mathrm{AdS}_{4}\right): \\
& T_{u u}=\frac{3 M \alpha^{3}}{8 \pi G_{N} R\left(u^{2}+\alpha^{2}\right)^{\frac{5}{2}} \sqrt{v^{2}+\alpha^{2}}}, \quad T_{u v}=\frac{M \alpha^{3}}{8 \pi G_{N} R\left(u^{2}+\alpha^{2}\right)^{\frac{3}{2}}\left(v^{2}+\alpha^{2}\right)^{\frac{3}{2}}}, \\
& T_{v v}=\frac{3 M \alpha^{3}}{8 \pi G_{N} R\left(v^{2}+\alpha^{2}\right)^{\frac{5}{2}} \sqrt{u^{2}+\alpha^{2}}}, \quad T_{\theta \theta}=\frac{3 M \alpha^{3}(u-v)^{2}}{8 \pi G_{N} R\left(u^{2}+\alpha^{2}\right)^{\frac{3}{2}}\left(v^{2}+\alpha^{2}\right)^{\frac{3}{2}}}, \\
& d=4\left(\operatorname{AdS}_{5}\right): \\
& T_{u u}=\frac{M \alpha^{4}}{\pi G_{N} R\left(\alpha^{2}+u^{2}\right)^{3}\left(\alpha^{2}+v^{2}\right)}, T_{u v}=\frac{M \alpha^{4}}{2 \pi G_{N} R\left(\alpha^{2}+u^{2}\right)^{2}\left(\alpha^{2}+v^{2}\right)^{2}}, \\
& T_{v v}=\frac{M \alpha^{4}}{\pi G_{N} R\left(\alpha^{2}+u^{2}\right)\left(\alpha^{2}+v^{2}\right)^{3}}, \\
& T_{\theta \theta}=\frac{M \alpha^{4}(u-v)^{2}}{4 \pi G_{N} R\left(\alpha^{2}+u^{2}\right)^{2}\left(\alpha^{2}+v^{2}\right)^{2}}, \quad T_{\phi \phi}=\frac{M \alpha^{4} \sin ^{2} \theta(u-v)^{2}}{4 \pi G_{N} R\left(\alpha^{2}+u^{2}\right)^{2}\left(\alpha^{2}+v^{2}\right)^{2}} \text {. }
\end{aligned}
$$

The angular coordinates $\theta$ for $d=3$ and $(\theta, \phi)$ for $d=4$ are those of the polar coordinates of $R^{2}: d \rho^{2}+\rho^{2} d \theta^{2}$ and $R^{3}: d \rho^{2}+\rho^{2}\left(d \theta^{2}+\sin ^{2} \theta d \phi^{2}\right)$, respectively. For $d=4$, the above expression agrees with the one in [21].

Note that we can easily confirm the traceless condition

$$
T_{a b} \eta^{a b}=0
$$


and the conservation law

$$
\partial_{a} T^{a b}=0
$$

In particular, the energy density $T_{t t}$ in each dimension is given by

$$
\begin{aligned}
& d=2: \quad T_{t t}=\frac{M \alpha^{2}}{4 \pi G_{N} R} \cdot \frac{\left(t^{2}+x^{2}+\alpha^{2}\right)^{2}+4 t^{2} x^{2}}{\left(\left(x^{2}-t^{2}-\alpha^{2}\right)^{2}+4 x^{2} \alpha^{2}\right)^{2}}, \\
& d=3: \quad T_{t t}=\frac{M \alpha^{3}}{\pi G_{N} R} \cdot \frac{\left(\rho^{2}+t^{2}+\alpha^{2}\right)^{2}+2 \rho^{2} t^{2}}{\left(\left(\rho^{2}-t^{2}-\alpha^{2}\right)^{2}+4 \alpha^{2} \rho^{2}\right)^{\frac{5}{2}}}, \\
& d=4: \quad T_{t t}=\frac{M \alpha^{4}}{\pi G_{N} R} \cdot \frac{3\left(\rho^{2}+t^{2}+\alpha^{2}\right)^{2}+4 \rho^{2} t^{2}}{\left(\left(\rho^{2}-t^{2}-\alpha^{2}\right)^{2}+4 \alpha^{2} \rho^{2}\right)^{3}} .
\end{aligned}
$$

By using these expressions we can confirm

$$
\int d^{d-1} x T_{t t}=\frac{m R}{\alpha}=E,
$$

for any $d$, by using the relation (2.14). Therefore the total energy agrees with that of the particle (2.5) as expected.

To understand how the excitations propagate, it is useful to look at the time evolution of $T_{t t}$. This is plotted in Fig, We can observe a peak on the light-cone $t^{2}=\rho^{2}$ as can be understood as the light-like propagation (or shock waves as in [21]) of the initial excitations at $t=\rho=0$.

The parameter $\alpha$ parameterizes the width of this peak and therefore it measures the size of the localized excitations. Notice that for $d=2$, the heights of the two peaks are equal and stay constant due to the total energy conservation. We sketched the essence of this behavior in Fig 4 for $d=2$. In the zero width limit $\alpha \rightarrow 0$ we find $T_{t t}$ gets delta-functionally localized:

$$
\begin{aligned}
& d=2: \quad T_{t t} \rightarrow \frac{E}{2}(\delta(t+\rho)+\delta(t-\rho)), \\
& d=3: \quad T_{t t} \rightarrow \frac{E}{2 \pi \rho}(\delta(t+\rho)+\delta(t-\rho)), \\
& d=4: \quad T_{t t} \rightarrow \frac{E}{4 \pi \rho^{2}}(\delta(t+\rho)+\delta(t-\rho)) .
\end{aligned}
$$

\section{Perturbative Analysis of Holographic Entanglement Entropy under Local Quenches}

The entanglement entropy $S_{A}$ is defined as the von-Neumann entropy when we trace out the subsystem $B$, which is the complement of $A$. The subsystem $A$ is an arbitrary chosen spacelike region on a time slice. Therefore, in a time-dependent background, $S_{A}$ depends on the time even if we fix the shape of the region $A$. This is expected to be an important quantity which 

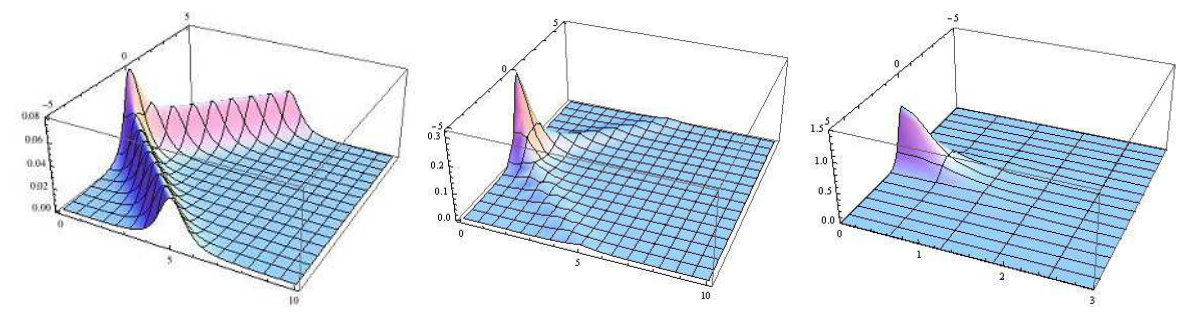

Figure 3: The profiles of the energy density $T_{t t}$ for $d=2$ (left), $d=3$ (middle) and $d=4$ (right) as a function of $\rho$ and $t$ within the range $-5<t<5$. We set $\alpha=R=M=G_{N}=1$.

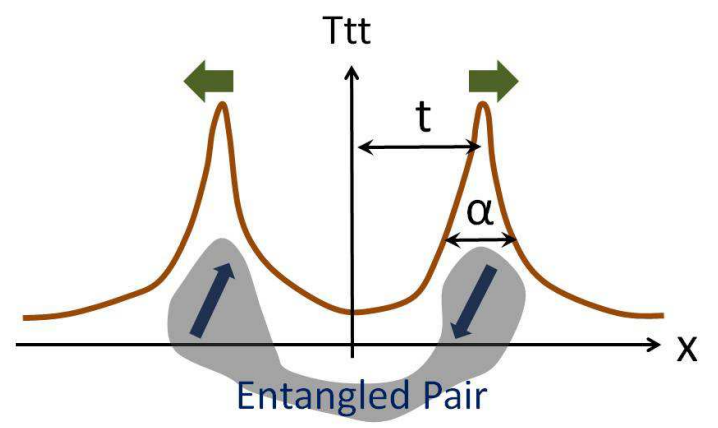

Figure 4: A sketch of time evolution of energy density and entangled pairs for $d=2$. The understanding of detailed structure of quantum entanglement and entangled pairs is the main subject of this paper as will be studied in later sections.

characterizes various non-equilibrium processes in quantum many-body systems such as quantum quenches [6, 8, 7].

In holographic setups, we can calculate $S_{A}$ by using the holographic entanglement entropy (HEE). In $d+1$ dimensional static gravity backgrounds, we can calculate $S_{A}$ by the formula [16]

$$
S_{A}=\frac{\operatorname{Area}\left(\gamma_{A}\right)}{4 G_{N}}
$$

where $\gamma_{A}$ is the $d-1$ dimensional minimal area surface on a time slice which ends on the boundary of $A$ i.e. $\partial A$. For spherically symmetric subsystems, we can manifestly prove (3.1) in pure AdS spaces via the bulk to boundary relation [10] as shown in [27]. In time-dependent backgrounds, there is no natural time-slice in the bulk AdS and we need to employ the covariant version of HEE [17]. This is given by redefining $\gamma_{A}$ to be an extremal surface in the Lorentzian spacetime.

Below we would like to compute the holographic entanglement entropy in the gravity dual of 
the local quench obtained from the coordinate transformation of the back hole solution (2.13) as explained in the previous section. In this section we perform a perturbative calculation assuming that the back-reaction from the particle is very small, keeping only the leading term proportional to $M$. This allows us a relatively simple calculation which is applicable to any dimension $d$.

\subsection{Perturbative Calculations of HEE under Local Quenches}

Consider the first order perturbation of the metric

$$
g_{\mu \nu}=g_{\mu \nu}^{(0)}+g_{\mu \nu}^{(1)}+O\left(M^{2}\right)
$$

where $g_{\mu \nu}^{(0)}$ represents the metric of pure $\operatorname{AdS}_{d+1}(\underline{2.1}) ; g_{\mu \nu}^{(1)}$ is the leading perturbation due to the back-reaction, which is of order $M$ in our case. We obtained $g_{\mu \nu}^{(1)}$ from the direct calculation explained in the previous section, though we will not write its complicated expression explicitly.

If we know the extremal surface $\gamma_{A}$ in the pure $\operatorname{AdS}_{d+1}$, then the perturbed area of an extremal surface can be found as

$$
\Delta \text { Area }=\frac{1}{2} \int d^{d-1} \xi \sqrt{G^{(0)}} \operatorname{Tr}\left[G^{(1)}\left(G^{(0)}\right)^{-1}\right] .
$$

Here $G$ is the induced metric on the surface $\gamma_{A}$ :

$$
G_{\alpha \beta}^{(0)}=\frac{\partial X^{\mu}}{\partial \xi^{\alpha}} \frac{\partial X^{\nu}}{\partial \xi^{\beta}} g_{\mu \nu}^{(0)}, \quad G_{\alpha \beta}^{(1)}=\frac{\partial X^{\mu}}{\partial \xi^{\alpha}} \frac{\partial X^{\nu}}{\partial \xi^{\beta}} g_{\mu \nu}^{(1)},
$$

where $\xi^{\alpha}(\alpha=1,2, \cdots, d-1)$ is the coordinate of the codimension two surface $\gamma_{A}$. The embedding function $X^{\mu}(\xi)$ is that of $\gamma_{A}$ in the pure $\operatorname{AdS}_{d+1}$. Notice the useful fact that we do not need to know the precise shape of $\gamma_{A}$ in the perturbed metric to calculate (3.3).

\subsection{Explicit Calculations of HEE under Local Quenches}

Consider the $d=3$ case $\left(\mathrm{AdS}_{4}\right)$ first. We take the subsystem $A$ to be a round disk with the radius $l$, defined by $x_{1}^{2}+x_{2}^{2} \leq l^{2}$. The corresponding extremal surface (or equally minimal surface) is given by the half sphere [16]

$$
z=\sqrt{l^{2}-x_{1}^{2}-x_{2}^{2}}
$$

We can take $\left(\xi^{1}, \xi^{2}\right)=\left(x_{1}, x_{2}\right)$ and then we find that the area density is given by

$$
\frac{1}{2} \sqrt{\tilde{G}^{(0)}} \operatorname{Tr}\left[\tilde{G}^{(1)}\left(\tilde{G}^{(0)}\right)^{-1}\right]=\frac{4 M R^{2} \rho^{2}}{l\left(e^{2 \beta} R^{4}+e^{-2 \beta}\left(l^{2}-t^{2}\right)^{2}+2 R^{2}\left(2 \rho^{2}+t^{2}-l^{2}\right)\right)^{3 / 2}},
$$

where $\rho=\sqrt{\sum_{i=1}^{d-1} x_{i}^{2}}$. 
The area perturbation is found by integrating (3.6) over $x$ and $y$. By plugging this into (3.1) we obtain the increased amount of HEE, denoted by $\Delta S_{A}$, compared with $S_{A}$ for the ground state dual to the pure AdS:

$$
\Delta S_{A}=\frac{\pi M}{4 G_{N} R \alpha l}\left(\frac{l^{4}-2 l^{2} t^{2}+\left(\alpha^{2}+t^{2}\right)^{2}}{\sqrt{l^{4}+2 l^{2}\left(\alpha^{2}-t^{2}\right)+\left(\alpha^{2}+t^{2}\right)^{2}}}-\left|t^{2}+\alpha^{2}-l^{2}\right|\right),
$$

where we used the relation (2.12). Note that the area law divergence [28] is canceled in $\Delta S_{A}$ because $\Delta S_{A}$ is defined from $S_{A}$ by subtracting the entanglement entropy for the ground state. For example, at $t=0$ we find

$$
\left.\Delta S_{A}\right|_{t=0}=\frac{\pi M l^{3}}{2 G_{N} R \alpha\left(l^{2}+\alpha^{2}\right)} \quad(l \leq \alpha),\left.\quad \Delta S_{A}\right|_{t=0}=\frac{\pi M \alpha^{3}}{2 G_{N} R l\left(l^{2}+\alpha^{2}\right)} \quad(l>\alpha),
$$

The time-evolution of (3.7) is plotted in Fig 5. Notice that $\Delta S_{A}$ respects the time-reversal symmetry. When $l>\alpha$, the entanglement entropy initially grows with the time for $t>0$ and reaches the maximum at $t=\sqrt{l^{2}-\alpha^{2}}$ as in the left graph in Fig 5. Later, it decreases as

$$
\Delta S_{A} \simeq \frac{\pi M \alpha^{3} l^{3}}{2 G_{N} R t^{6}}
$$

When $l<\alpha$, the entanglement entropy always decreases for $t>0$. At late time we have the behavior (3.9). Notice also that the width $\Delta t$ of the peak around $t \simeq \sqrt{l^{2}-\alpha^{2}}$ is estimated as $\Delta t \sim \alpha$ when $\alpha<<l$. These results can be intuitively understood because at $t=\sqrt{l^{2}-\alpha^{2}}$ the particle in AdS passes through the minimal surface (3.5). Refer to the Fig 2 again. In the dual CFT, they can be naturally understood if we remember the excitations propagate at the speed of light as we will discuss later.

Notice also an interesting property that the height of the peak at $t=\sqrt{l^{2}-\alpha^{2}}$ stays constant, given by $S_{A}=\frac{\pi M}{4 G_{N} R}$, and thus does not decrease under the time-evolution. This can also be seen from the right graph in Fig.5. This is rather different from the behavior of energy stress tensor studied in the previous section (see Fig, 3).

It is also intriguing to shift the center of the subsystem $A$ relative to the trajectory of the falling particle in the $\left(x_{1}, y_{2}\right)$ plane. We take the minimal surface in the pure AdS to be

$$
z=\sqrt{l^{2}-\left(x_{1}-\xi\right)^{2}-x_{2}^{2}}
$$

where $\xi$ is the distance between the center of $A$ and the falling particle.

We plotted the results of $\Delta S_{A}$ in Fig 6 for different values of $\xi$. In general one may notice that the peak is broadened so that it is spread over $l-\xi<t<l+\xi$. In the gravity dual, this is easily explained from the propagation of gravitational waves from the falling particle. This also qualitatively agrees with the results of local quenches in two dimensional CFTs [8]. 

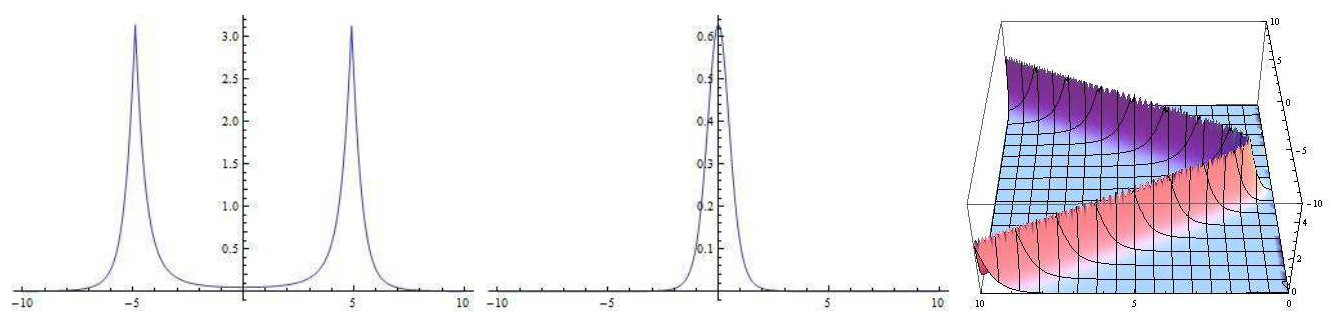

Figure 5: The plots of $\Delta S_{A}$ at $\xi=0$ for $d=3$. The left and middle one describe $S_{A}$ as a function of $t$. We choose $(\alpha, l)=(1,5)$ (left) and $(\alpha, l)=(1,0.5)$ (middle), respectively. The right $3 \mathrm{~d}$ graph expresses $S_{A}$ as a function of $t$ and $l$ for $\alpha=1$. The horizontal coordinate corresponds to $l$. We took the range $-10<t<10$ and $0<l<10$. We set $R=4 G_{N}=M=1$.
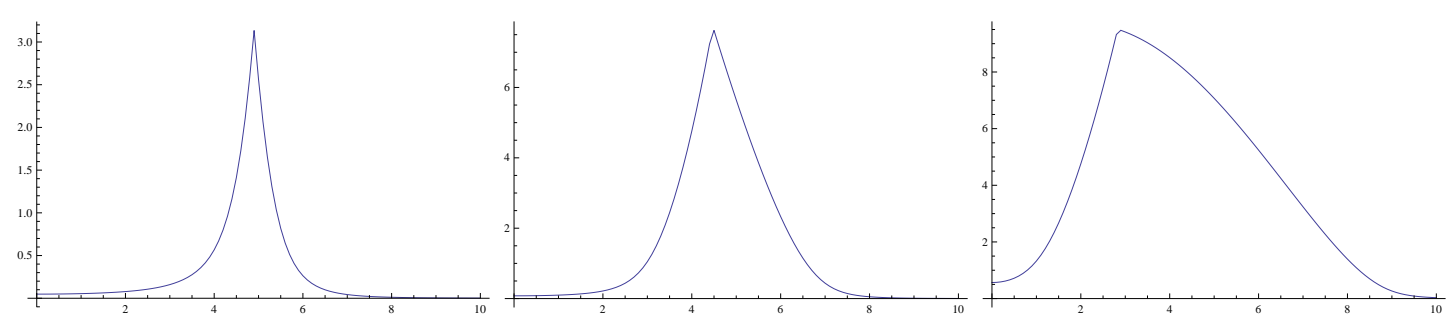

Figure 6: The plots of $\Delta S_{A}$ for $d=3$ as a function of time $t$. The left, middle and right graph correspond to $(\alpha, l, \xi)=(1,5,0),(1,5,2)$ and $(1,5,4)$, respectively. We set $R=4 G_{N}=M=1$.

It is straightforward to generalize the above results to other dimensions. In $d=2$ and $d=4$, the expression of $\Delta S_{A}$ (3.7) for $d=3$ is replaced with 2

$$
\begin{aligned}
& d=2: \quad \Delta S_{A}=\frac{2 M l \alpha+M\left(l^{2}-\alpha^{2}-t^{2}\right) \arctan \left(\frac{2 \alpha l}{t^{2}+\alpha^{2}-l^{2}}\right)}{8 G_{N} l R \alpha}, \\
& d=4: \quad \Delta S_{A}=\frac{\pi M}{8 G_{N} l R \alpha}\left(\frac{1}{\frac{2 \alpha l}{\left(\alpha^{2}-l^{2}+t^{2}\right)^{2}}+\frac{1}{2 \alpha l}}-3\left(\alpha^{2}-l^{2}+t^{2}\right) \arctan \left(\frac{2 \alpha l}{\alpha^{2}-l^{2}+t^{2}}\right)+4 \alpha l\right) .
\end{aligned}
$$

We can confirm that the behaviors of $\Delta S_{A}$ in $d=2$ and $d=4$ are very similar to that in $d=3$ and thus we will not show them the explicitly.

At late time $t>>l$, they are approximated by

$$
\begin{aligned}
& d=2: \quad \Delta S_{A} \simeq \frac{M \alpha^{2} l^{2}}{3 G_{N} R t^{4}}, \\
& d=4: \quad \Delta S_{A} \simeq \frac{8 \pi \alpha^{4} l^{4} M}{5 G_{N} R t^{8}} .
\end{aligned}
$$

This shows that $\Delta S_{A}$ decays like $\sim l^{d} \cdot t^{-2 d}$ at late time. Notice that the perturbative calculation is always justified at late time as the back-reaction to $\gamma_{A}$ for a finite $l$ clearly gets suppressed.

\footnotetext{
${ }^{2}$ In $d=2$ the subsystem is chosen to be an interval $-l \leq x \leq l$.
} 


\subsection{Small Subsystem Limit: An Analogue of the first law of thermodynamics}

In the small limit of the subsystem size $l$, we can trust the perturbative results of $\Delta S_{A}$ (3.7) and (3.11). This is because the surface $\gamma_{A}$ is situated near the AdS boundary and therefore the deviation of the metric from the pure AdS is very small [29]. In asymptotically AdS backgrounds which are static and translation invariant, a relation which looks like the first law of thermodynamics has been found in the small size limit of $A$ [29]:

$$
T_{e f f} \cdot \Delta S_{A}=\Delta E_{A},
$$

where $E_{A}$ is the energy in the subsystem $A$ given by $E_{A}=\int_{A} d x^{d-1} T_{t t}$. The effective temperature is defined by $T_{\text {eff }}=\frac{c_{A}}{l}$, where the constant $c_{A}$ only depends on the shape of the subsystem $A$ and is independent from the details of the CFT we consider. When $A$ is a $d-1$ dimensional ball with the radius $l$ as we choose in this paper, we have

$$
T_{e f f}=\frac{d+1}{2 \pi l} .
$$

Thus it is intriguing to see if this relation (3.13) holds in our time-dependent and inhomogeneous setup of local quenches. We can calculate the energy density from (2.24), and $\Delta S_{A}$ from (3.7) and (3.11) in the limit $l<<\sqrt{\alpha^{2}+t^{2}}$ as follows:

$$
\begin{aligned}
& d=2: \quad T_{t t}=\frac{M \alpha^{2}}{4 \pi G_{N} R\left(t^{2}+\alpha^{2}\right)^{2}}, \quad \Delta S_{A}=\frac{M \alpha^{2} l^{2}}{3 G_{N} R\left(t^{2}+\alpha^{2}\right)^{2}} \\
& d=3: \quad T_{t t}=\frac{M \alpha^{3}}{\pi G_{N} R\left(t^{2}+\alpha^{2}\right)^{3}}, \quad \Delta S_{A}=\frac{\pi \alpha^{3} l^{3}}{2 G_{N} R\left(t^{2}+\alpha^{2}\right)^{3}} \\
& d=4: \quad T_{t t}=\frac{3 M \alpha^{4}}{\pi G_{N} R\left(t^{2}+\alpha^{2}\right)^{4}}, \quad \Delta S_{A}=\frac{8 \pi M \alpha^{4} l^{4}}{5 G_{N} R\left(t^{2}+\alpha^{2}\right)^{4}} .
\end{aligned}
$$

By using these expressions we can explicitly confirm the relation (3.13) for any $d$.

\subsection{Large Subsystem Limit}

Before we go on, we would like to write down the result in the large subsystem limit $l>>\sqrt{t^{2}+\alpha^{2}}$. In this limit, we find from (3.7) and (3.11):

$$
\begin{aligned}
& d=2: \quad \Delta S_{A}=\frac{8 \alpha^{2}}{3 l^{2}} m R, \\
& d=3: \quad \Delta S_{A}=\frac{\pi \alpha^{3}}{l^{3}} m R, \\
& d=4: \quad \Delta S_{A}=\frac{64 \alpha^{4}}{5 l^{4}} m R .
\end{aligned}
$$

Note that these are time-independent. 


\section{Exact Holographic Entanglement Entropy for 2d Local Quenches}

We can actually find exact extremal surfaces (i.e. geodesics) in the $\mathrm{AdS}_{3}$ case $(d=2)$. We take the subsystem $A$ to be an interval at a constant time in the dual $\mathrm{CFT}_{2}$. In the holographic calculation, first we obtain a geodesic in the metric (2.13), which is asymptotically global $\mathrm{AdS}_{3}$. Then we map it into an asymptotically Poincare AdS metric by the coordinate transformation (2.10).

Initially, we will assume $M-R^{2}<0$ and thus the geometry (2.13) has the deficit angle at $r=0$. If we were allowed to take $\theta$ to be $2 \pi \frac{R}{\sqrt{R^{2}-M}}$ instead of $2 \pi$, the geometry gets smooth. Later we will come back to the case $M-R^{2}>0$ i.e. the BTZ black hole.

In the near AdS boundary limit, the map between the point $\left(\tau_{\infty}, \theta_{\infty}, r_{\infty}\right)$ in (2.13) and the point $\left(t, z_{\infty}, x_{\infty}\right)$ in the Poincare AdS is given by 3

$$
\begin{aligned}
& \tan \tau_{\infty}=\frac{2 R t}{\left.R^{2} e^{\beta}+e^{-\beta}\left(x_{\infty}^{2}-t^{2}\right)\right)}, \\
& \tan \theta_{\infty}=-\frac{2 R x_{\infty}}{e^{-\beta}\left(x_{\infty}^{2}-t^{2}\right)-R^{2} e^{\beta}}, \\
& r_{\infty}=\frac{1}{z_{\infty}} \sqrt{R^{2} x_{\infty}^{2}+\frac{1}{4}\left(e^{-\beta}\left(x_{\infty}^{2}-t^{2}\right)-R^{2} e^{\beta}\right)^{2}} .
\end{aligned}
$$

Notice that $z_{\infty}$ is interpreted as the UV cut off (or lattice spacing) in the dual CFT. In the above expressions, we chose the range of $\tau_{\infty}$ and $\theta_{\infty}$ to be $[-\pi, \pi]$.

We can specify the geodesic $\gamma_{A}$ in (2.13) by

$$
\tau=\tau(\theta), \quad r=r(\theta)
$$

Its length $\left|\gamma_{A}\right|$ reads

$$
\left|\gamma_{A}\right|=\int d \theta \sqrt{r^{2}+\frac{R^{2}}{r^{2}+R^{2}-M} r^{\prime 2}-\left(r^{2}+R^{2}-M\right) \tau^{\prime 2}}
$$

The minimal length condition is summarized as

$$
\begin{aligned}
& \frac{d \tau}{d \theta}=\frac{A r^{2}}{r^{2}+R^{2}-M}, \\
& \frac{d r}{d \theta}=\frac{r}{R} \sqrt{A^{2} r^{2}+\left(B^{2} r^{2}-1\right)\left(r^{2}+R^{2}-M\right)},
\end{aligned}
$$

where $A$ and $B$ are integration constants.

\subsection{Symmetric Intervals}

Consider the case where the subsystem $A$ is given by an interval $-l \leq x \leq l$ at time $t$. The excitations of the local quench occur at $x=0$ because the falling particle is situated at $x=0$. In

\footnotetext{
${ }^{3}$ To fix the signs of $\tau_{\infty}$ and $\theta_{\infty}$, we need to go back to the original coordinate transformation (2.10).
} 
the global AdS, this is mapped into an interval $-\theta_{\infty} \leq \theta \leq \theta_{\infty}$ at a constant time slice $\tau=\tau_{\infty}$, where we can assume $0<\theta_{\infty}<\pi$ without losing generality (see Fig[7). Since we can assume $\tau$ is constant on $\gamma_{A}$, the extremal surface condition (4.4) gets simplified to

$$
\frac{d r}{d \theta}=\frac{r}{R r_{*}} \sqrt{\left(r^{2}+R^{2}-M\right)\left(r^{2}-r_{*}^{2}\right)}
$$

where $r=r_{*}$ is an integration constant and $r_{*}$ is the minimum value of $r$ on $\gamma_{A}$, i.e. the turning point.

\subsubsection{Case 1: $M \leq R^{2}$}

Let us first assume $0<\theta_{\infty}<\frac{\pi}{2}$. The curve $\gamma_{A}$ with the minimum length is given by the geodesic which takes the angle range $-\theta_{\infty} \leq \theta<\theta_{\infty}$ (see the left picture of Fig:7). The point $\theta=0$ corresponds to the turning point $r=r_{*}$. Thus we find 4

$$
\begin{aligned}
\theta_{\infty} & =\int_{r_{*}}^{\infty} d r \frac{R r_{*}}{r \sqrt{\left(r^{2}+R^{2}-M\right)\left(r^{2}-r_{*}^{2}\right)}} \\
& =\frac{R}{2 \sqrt{R^{2}-M}}\left[\frac{\pi}{2}+\arcsin \left(\frac{R^{2}-M-r_{*}^{2}}{R^{2}-M+r_{*}^{2}}\right)\right] .
\end{aligned}
$$

It is useful to rewrite this into

$$
\cos \left(\frac{2 \sqrt{R^{2}-M}}{R} \theta_{\infty}\right)=\frac{r_{*}^{2}-R^{2}+M}{r_{*}^{2}+R^{2}-M} .
$$

Finally, the HEE is given by

$$
\begin{aligned}
S_{A} & =\frac{R}{2 G_{N}} \int_{r_{*}}^{r_{\infty}} d r \frac{r}{\sqrt{\left(r^{2}+R^{2}-M\right)\left(r^{2}-r_{*}^{2}\right)}} \\
& =\frac{R}{2 G_{N}} \log \frac{2 r_{\infty}}{\sqrt{R^{2}-M+r_{*}^{2}}} .
\end{aligned}
$$

In the case $\theta_{\infty}>\frac{\pi}{2}$, on the other hand, the curve $\gamma_{A}$ is given by the geodesic which takes the angular range $\theta_{\infty}<|\theta|<\pi$ (see the right picture of Fig,7). Thus its turning point is at $\theta=\pi$. This guarantees the basic property of entanglement entropy written as $S_{A}=S_{B}$ for pure states, where $B$ is the complement of $A$. Thus for $\theta_{\infty}>\frac{\pi}{2}$, we find the correct HEE from (4.8) by replacing $\theta_{\infty}$ with $\pi-\theta_{\infty}$ in (4.7).

\subsubsection{Case 2: $M>R^{2}$}

For $M>R^{2}$, the geometry (2.13) becomes the BTZ black hole. In this case, the basic result can be obtained from the previous one by the analytic continuation $\sqrt{R^{2}-M} \rightarrow i \sqrt{M-R^{2}}$ : the relation

\footnotetext{
${ }^{4}$ In this paper, the function $\arcsin (x)$ takes values between $-\frac{\pi}{2}$ and $\frac{\pi}{2}$.
} 

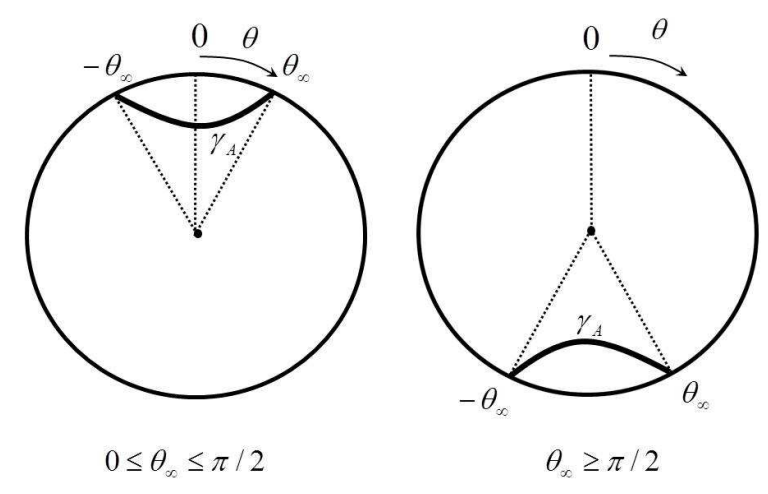

Figure 7: The sketch of $\gamma_{A}$ in the asymptotically global AdS background (2.13). The left and right picture corresponds to $0 \leq \theta_{\infty} \leq \pi / 2$ and $\theta_{\infty} \geq \pi / 2$, respectively.

(4.7) is replaced with

$$
\cosh \left(\frac{2 \sqrt{M-R^{2}}}{R} \theta_{\infty}\right)=\frac{r_{*}^{2}-R^{2}+M}{r_{*}^{2}+R^{2}-M} .
$$

For $0<\theta_{\infty}<\frac{\pi}{2}$, the holographic entanglement entropy $S_{A}$ can be found from the same expression (4.8), which is denoted by $S_{A}\left(\theta_{\infty}\right)$. For $\theta_{\infty}>\frac{\pi}{2}, S_{A}$ is given by $S_{A}\left(\pi-\theta_{\infty}\right)$ as in the $M<R^{2}$ case.

In this calculation we implicitly assume that we are considering a star solution and that outside of the star is described by the BTZ black hole solution (2.13). Thus this is dual to a pure state in the CFT and indeed our construction satisfies $S_{A}=S_{B}$.

\subsubsection{Final Results}

The final result is plotted in Fig 8 , For a small $M$ the exact result nicely agrees with the one from the perturbation theory (3.11). Even for a large $M$, the agreement is very good except the peak at $t \sim \sqrt{l^{2}-\alpha^{2}}$. Indeed, we can analytically show that in both the limit $t \rightarrow 0$ and $t=\infty, S_{A}$ approaches to

$$
S_{A}(0)=S_{A}(t=\infty)=\frac{c}{3} \log \frac{2 l}{z_{\infty}},
$$

where we employed the well-known relation $c=\frac{3 R}{2 G_{N}}[30$. This reproduces the well-known result of the entanglement entropy for ground states in CFTs [31, 32, 2]. Remember that $z_{\infty}$ corresponds to the UV cut off (lattice spacing) in the dual CFTs. 

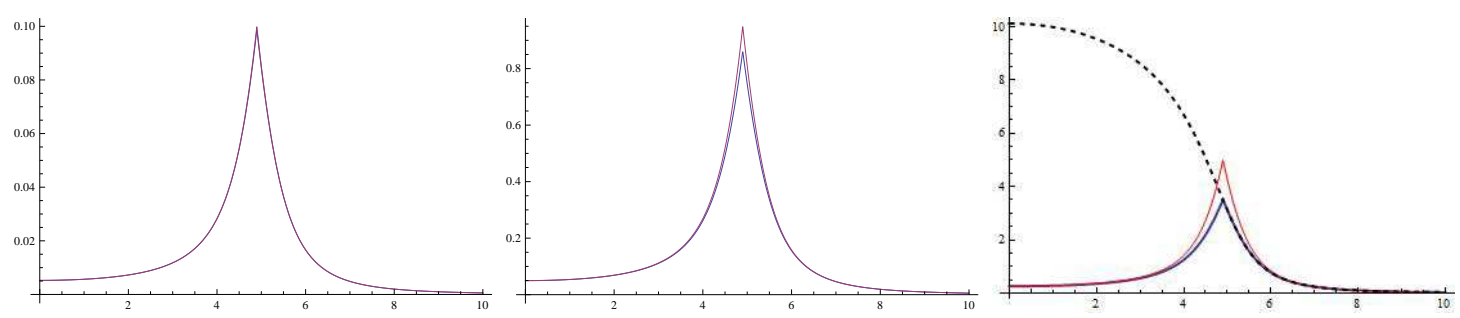

Figure 8: The exact plots of $\Delta S_{A}$ as a function of time $t(>0)$. The left, middle and right graph correspond to $M=0.1,0.95,5$, respectively. The blue and red curve correspond to the result from the exact analysis and perturbation theory, respectively. They coincide almost completely for $M=0.1$. The dotted black one for $M=5$ corresponds to the thermal local quench given by (4.11). We set $R=\alpha=4 G_{N}=1$ and $l=5$.

\subsubsection{Thermal Local Quenches}

In the previous analysis for $M>R^{2}$, we obtained $S_{A}$ by replacing a black hole with a star in order to have a gravity dual of a pure state, which is usually assumed in the study of quantum quenches. If we deal with a falling black hole instead of a massive star, we will obtain $S_{A}$ as follows

$$
S_{A}=\min \left\{S_{A}\left(\theta_{\infty}\right), S_{A}\left(\pi-\theta_{\infty}\right)+\frac{\pi \sqrt{M-R^{2}}}{2 G_{N}}\right\},
$$

where $\min \{a, b\}$ denotes the smaller one among $a$ and $b$. The term $\frac{\pi \sqrt{M-R^{2}}}{2 G_{N}}$ corresponds to a half of black hole entropy and arises because $\gamma_{A}$ wraps a half of horizon. The topological condition of $\gamma_{A}$ of HEE requires that the subsystem $A$ should be homologous to $\gamma_{A}$. Therefore in the presence of the black hole horizon, we cannot simply change the geodesic from the one passing through $\theta=0$ to the one passing through $\theta=\pi$. If we want to deform $\gamma_{A}$ in this way, we need also to wrap $\gamma_{A}$ on the horizon as its disconnected part. We plotted $S_{A}$ for this thermal local quench in the third graph of Fig, 8

\subsection{General Formulation}

Now let us extend the previous analysis to more general setups where the subsystem $A$ is given by an arbitrary interval $l^{(1)} \leq x \leq l^{(2)}$ at the time $t$. The surface $\gamma_{A}$ is defined by the geodesic curve whose two end points are given by $(x, t, z)=\left(l^{(i)}, t, z_{\infty}\right)(i=1,2)$ in the Poincare AdS3 and 
equally by $(\tau, \theta, r)=\left(\tau_{\infty}^{(i)}, \theta_{\infty}^{(i)}, r_{\infty}^{(i)}\right)$ in the global AdS. Note that their relations are given by:

$$
\begin{aligned}
& \tan \tau_{\infty}^{(i)}=\frac{2 R t}{R^{2} e^{\beta}+e^{-\beta}\left(\left(l^{(i)}\right)^{2}-t^{2}\right)}, \\
& \tan \theta_{\infty}^{(i)}=-\frac{2 R l^{(i)}}{e^{-\beta}\left(\left(l^{(i)}\right)^{2}-t^{2}\right)-R^{2} e^{\beta}}, \\
& r_{\infty}^{(i)}=\frac{1}{z_{\infty}} \sqrt{R^{2}\left(l^{(i)}\right)^{2}+\frac{1}{4}\left(e^{-\beta}\left(\left(l^{(i)}\right)^{2}-t^{2}\right)-R^{2} e^{\beta}\right)^{2}} .
\end{aligned}
$$

Then the HEE reads

$$
S_{A}=\frac{R}{4 G_{N}} \sum_{i=1}^{2}\left[\int_{r_{*}}^{r_{\infty}^{(i)}} d r \frac{B r}{\sqrt{A^{2} r^{2}+\left(B^{2} r^{2}-1\right)\left(r^{2}+R^{2}-M\right)}}\right] .
$$

By integrating (4.4) we find

$$
\begin{aligned}
& \left|\tau_{\infty}^{(2)}-\tau_{\infty}^{(1)}\right|=\frac{R}{\sqrt{R^{2}-M}}\left[\frac{\pi}{2}+\arcsin \left(\frac{B^{2}\left(M-R^{2}\right)+A^{2}-1}{\sqrt{\left(B^{2}\left(R^{2}-M\right)+A^{2}-1\right)^{2}+4 B^{2}\left(R^{2}-M\right)}}\right)\right], \\
& \left|\theta_{\infty}^{(2)}-\theta_{\infty}^{(1)}\right|=\frac{R}{\sqrt{R^{2}-M}}\left[\frac{\pi}{2}+\arcsin \left(\frac{B^{2}\left(R^{2}-M\right)+A^{2}-1}{\sqrt{\left(B^{2}\left(R^{2}-M\right)+A^{2}-1\right)^{2}+4 B^{2}\left(R^{2}-M\right)}}\right)\right] .
\end{aligned}
$$

If we assume $0<\left|\theta_{\infty}^{(2)}-\theta_{\infty}^{(1)}\right|<\pi$, the HEE is computed as follows:

$$
\begin{gathered}
S_{A}=\frac{R}{4 G_{N}}\left[\log \left(r_{\infty}^{(1)} \cdot r_{\infty}^{(2)}\right)+\log \left(\frac{4 B^{2}}{\sqrt{\left(B^{2}\left(R^{2}-M\right)+A^{2}-1\right)^{2}+4 B^{2}\left(R^{2}-M\right)}}\right)\right], \\
=\frac{R}{4 G_{N}}\left[\log \left(r_{\infty}^{(1)} \cdot r_{\infty}^{(2)}\right)+\log \frac{2 \cos \left[\frac{\sqrt{R^{2}-M}\left|\tau_{\infty}^{(2)}-\tau_{\infty}^{(1)}\right|}{R}\right]-2 \cos \left[\frac{\sqrt{R^{2}-M}\left|\theta_{\infty}^{(2)}-\theta_{\infty}^{(1)}\right|}{R}\right]}{R^{2}-M}\right]
\end{gathered}
$$

In the case $\Delta \theta_{\infty}=\left|\theta_{\infty}^{(2)}-\theta_{\infty}^{(1)}\right|>\pi$, we need to replace $\Delta \theta_{\infty} \rightarrow 2 \pi-\Delta \theta_{\infty}$ as we did in the previous subsection.

So far we assumed $M<R^{2}$. If $M>R^{2}$, then the solution (2.13) is a BTZ black hole without any deficit angle. However, our analytical calculation done before still holds via the analytical continuation as in the previous case.

\subsection{An Interval with An Excited End Point}

Especially, let us focus on the case $\left(l^{(1)}, l^{(2)}\right)=(0, l)$. In this case, one of the end points of $A$ i.e. $x=0$ is excited by the local quench. The result is plotted in Fig.9. It is easy to see that $\Delta S_{A}$ is 

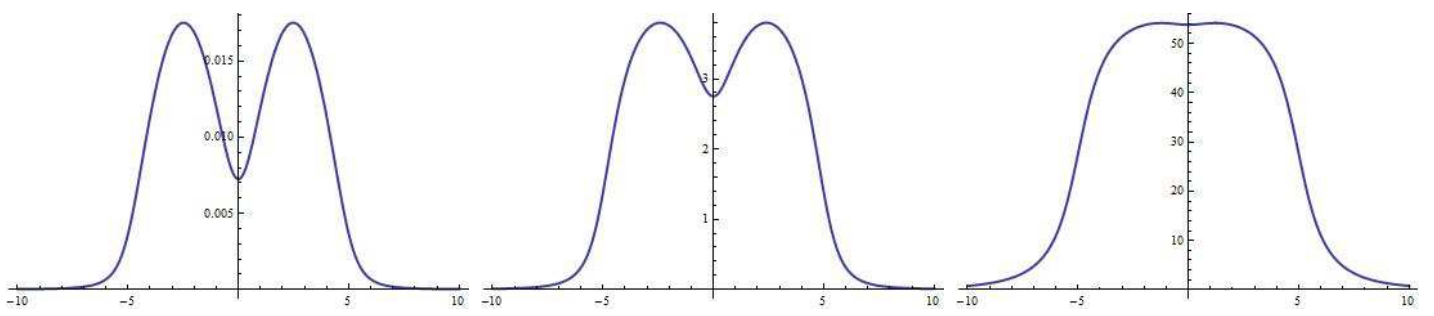

Figure 9: The exact plot of $\Delta S_{A}$ as a function of $t$ for $\left(l^{(1)}, l^{(2)}\right)=(0,5)$. The left, middle and right graph correspond to $M=0.01,5,500$, respectively. We set $R=\alpha=4 G_{N}=1$.

non-trivial during $-l \lesssim t \lesssim l$, as expected from the causality argument. This qualitatively agrees with the CFT result in [8]. As $M$ gets larger, the HEE looks like a step function.

In the late time limit $t \rightarrow \infty, S_{A}$ approaches to the result of the ground state

$$
S_{A}(t=\infty)=\frac{c}{3} \log \frac{l}{z_{\infty}}
$$

On the other hand, at the time $t=0$, we find the following result for $l>>\alpha$

$$
S_{A}(t=0)=S_{A}(t=\infty)+\frac{c}{6} \log \left(\frac{R^{2}}{R^{2}-M} \cdot \sin ^{2}\left(\frac{\pi \sqrt{R^{2}-M}}{2 R}\right)\right) .
$$

Notice that we always have $S_{A}(t=0) \geq S_{A}(t=\infty)$.

\subsubsection{Semi Infinite Limit $l \rightarrow \infty$}

It is useful to consider the limit $l>>t>>\alpha$ for $\left(l^{(1)}, l^{(2)}\right)=(0, l)$. We find

$$
\begin{aligned}
& \tau_{\infty}^{(1)} \simeq \pi-\frac{2 \alpha}{t}, \quad \tau_{\infty}^{(2)} \simeq \frac{2 t \alpha}{l^{2}} \\
& \theta_{\infty}^{(1)}=0, \quad \theta_{\infty}^{(2)} \simeq \pi-\frac{2 \alpha}{l} \\
& r_{\infty}^{(1)} \simeq \frac{R t^{2}}{2 \alpha z_{\infty}}, \quad r_{\infty}^{(2)} \simeq \frac{R l^{2}}{2 \alpha z_{\infty}}
\end{aligned}
$$

Finally the HEE is found to be

$$
\begin{aligned}
S_{A} & \simeq \frac{c}{3} \log \frac{R t l}{2 \alpha z_{\infty}}+\frac{c}{6} \log \left[\frac{4}{R^{2}-M} \cdot \sin \left(\frac{\sqrt{R^{2}-M}}{R}(\pi-\alpha / t)\right) \cdot \sin \left(\frac{\alpha \sqrt{R^{2}-M}}{R t}\right)\right] \\
& \simeq \frac{c}{3} \log \frac{l}{z_{\infty}}+\frac{c}{6} \log \frac{t}{\alpha}+\frac{c}{6} \log \left(\frac{R}{\sqrt{R^{2}-M}} \sin \left(\frac{\pi \sqrt{R^{2}-M}}{R}\right)\right) .
\end{aligned}
$$

In this way, we obtain the behavior

$$
S_{A}^{A d S} \sim \frac{c}{6} \log \frac{t}{\alpha}+\frac{c}{3} \log \frac{l}{z_{\infty}}
$$




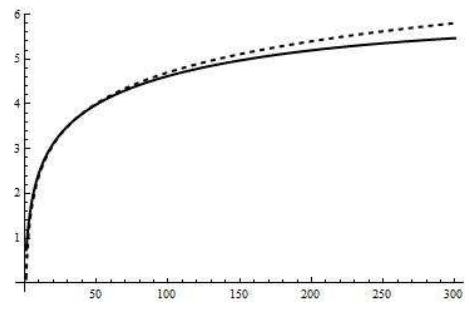

Figure 10: The approximation of $\Delta S_{A}$ by the function $\log t+$ const. for $\left(l^{(1)}, l^{(2)}\right)=(0,1000)$. The thick black curve is the plot of the exact result, while the dashed one is its approximation by $\log t+0.1$. We choose $M=0.5$ and set $R=\alpha=4 G_{N}=1$. They nicely agree with each other when $\alpha<<t<<l$.

for the time evolution. Refer to Fig 10 for the numerical confirmation.

On the other hand, the local quench induced by joining two half lines leads to the following behavior 8

$$
S_{A}^{\text {joint }} \sim \frac{c}{3} \log \frac{t}{z_{\infty}}+\frac{c}{6} \log \frac{l}{\epsilon},
$$

where $\epsilon$ is the cutoff for the process of local quench introduced in 8 , and is analogous to $\alpha$ in our model.

Note that both have the common important property that $S_{A}$ increases logarithmically after local quenches. This is a characteristic feature of local quenches in two dimension. To be more precise, the coefficient of $\log t$ is different between (4.20) and (4.21). Indeed, it is known that this coefficient depends on how we locally excite the system as found in the example analyzed in [7]. The difference of the coefficient in front of $\log l$ is easy to understands because in the case (4.20) the system was already joined before the quench, while in the other case (4.21), the system was originally disconnected. We will give a more detailed explanation of the two difference behaviors (4.20) and (4.21) in section 6.2 using the tensor network description.

\subsection{HEE for General Intervals}

Now we would like to turn to the HEE $S_{A}$ for a subsystem $A$ defined by a general interval $\left(l^{(1)}, l^{(2)}\right)$. Equally we can think that $A$ is parameterized by its width $l$ and the position of its center $\xi$ via

$$
l^{(1)}=\xi-l / 2, \quad l^{(2)}=\xi+l / 2 .
$$

We can explicitly calculate $S_{A}(l, \xi, t)$ by using the formula (4.15). We plotted $\Delta S_{A}$ as a function of $\xi$ for fixed values of $l$ and $t$ in Fig 11 .

First, we notice that for a small $l$ (the left graph of Fig,11), the initial peak at $\xi=0$ divides into two peaks under time evolution, keeping total sum of the heights conserved. This result looks 


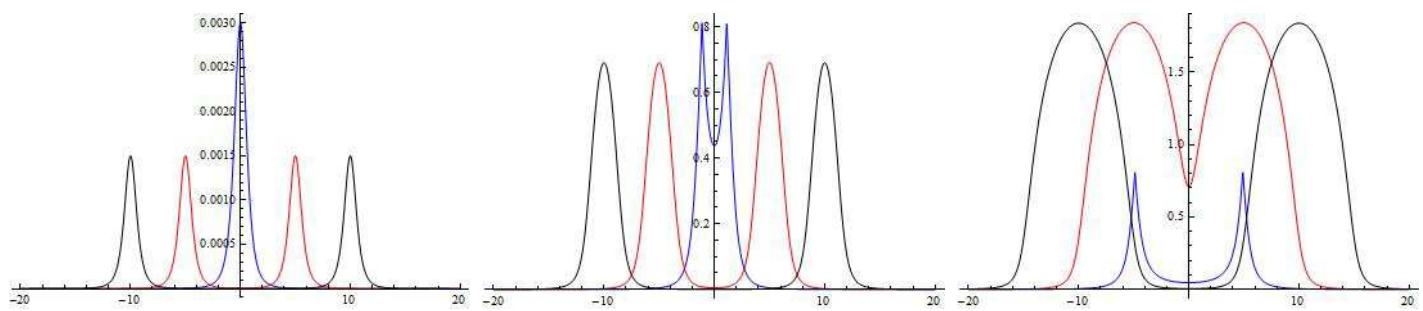

Figure 11: The plot of $\Delta S_{A}$ as a function of $\xi$. We set $l=0.1$ (left), $l=3$ (middle) and $l=10$ (right). In each graph, the blue, red and black curve describes $\Delta S_{A}(l, \xi, t)$ for $t=0, t=5$ and $t=10$, respectively. We choose $M=0.9$ and set $R=\alpha=4 G_{N}=1$.

very similar to the energy density $T_{t t}$ in two dimensional CFTs (see the left graph in Fig $[3$ ), where the energy conservation holds. Indeed, we can easily understand this coincidence from the first law relation (3.13) in the small $l$ limit, which can be rewritten as

$$
\Delta S_{A}(l, \xi, t) \simeq \frac{\pi l^{2}}{3} T_{t t}(\xi, t)
$$

Therefore we can conclude that in the small $l$ limit, the integral

$$
\int_{-\infty}^{\infty} d \xi \Delta S_{A}(l, \xi, t)
$$

does not depend on the time $t$.

Moreover, for $l>\alpha$, we find that $\Delta S_{A}$ at $t=0$ has a peak at $|\xi|=l / 2$. In the gravity dual, this is easy to understand because the geodesic $\gamma_{A}$ comes very close to the massive particle as explained in Fig,2, At a later time $t>l / 2$, the hump propagates at the speed of light, conserving its form. This occurs because $\gamma_{A}$ is now far from the falling particle and its shock waves propagates without changing its form. Therefore we expect that the integral (4.24) will approach to a constant value at late time $t>l / 2$. Indeed, we can confirm this numerically as shown in the Fig,12, This behavior roughly tells us that the long range entanglement will be generated at late time, while the short range one will be not. We will study more carefully such a dynamical structure of quantum entanglement in the next section, which will provide clear explanations for all of the results in this section.

\section{$5 \quad$ Entanglement Density}

In this section, we will introduce a new quantity which we call entanglement density. It can extract an essential structure of quantum entanglement from $S_{A}$ in two dimensional quantum field theories. We will apply this to our holographic local quenches in $\mathrm{AdS}_{3} / \mathrm{CFT}_{2}$. 


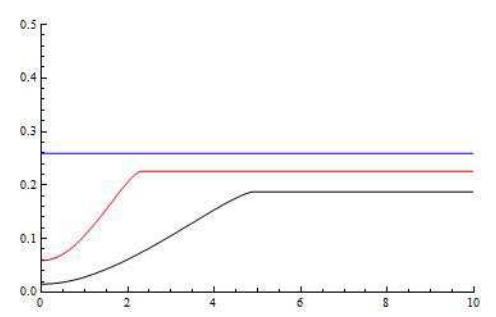

Figure 12: The numerical plot of $\frac{1}{l^{2}} \int d \xi \Delta S_{A}(l, \xi, t)$ as a function of time $t>0$. The blue, red and black graph correspond to $l=1,5,10$, respectively. In the small $l$ limit, this quantity should approach $\frac{\pi}{12} \simeq 0.262$ as follows from (4.23). We choose $M=0.5$ and set $R=\alpha=4 G_{N}=1$.

\subsection{Definition of Entanglement Density}

Consider a general two dimensional field theory in a certain pure state and suppose that we can calculate its entanglement entropy $S_{A}$. The subsystem $A$ is defined by the interval $l^{(1)} \leq x \leq l^{(2)}$ (or more simply $\left.\left[l^{(1)}, l^{(2)}\right]\right)$, where $x$ is the space coordinate of the field theory. Just for convenience, we will treat $l^{(1)}$ and $l^{(2)}$ to be independent parameters for a while. We would like to ask how to extract a structure of quantum entanglement explicitly from the entanglement entropy $S_{A}$ for arbitrary intervals.

For this purpose, we take into account only the two body entanglement and define the entanglement density $n(l, \xi, t)$ such that this counts the number of entangling pairs (or bits) between the two points $x=\xi-l / 2\left(=l^{(1)}\right)$ and $x=\xi+l / 2\left(=l^{(2)}\right)$. Imagine that we discretize the field theory into a lattice theory such as spin chains. In each point, we assume that there are several spins. Then $n(l, \xi, t)$ counts the number of entangled pairs of spins which are located at $x=\xi-l / 2$ and $x=\xi+l / 2$. Therefore the parameter $l$ describes the range of the entanglement and $\xi$ does the position of the center of the entangled pair.

Now we approximate $S_{A}$ by summing all entangling pair as follows (see Fig 13):

$$
S_{A}=\int_{l^{(1)}}^{l^{(2)}} d x\left[\int_{x-l^{(1)}}^{\infty} d w n(w, x-w / 2, t)+\int_{l^{(2)}-x}^{\infty} d w n(w, x+w / 2, t)\right] .
$$

By taking a derivative with respect to $l^{(2)}$ with $l^{(1)}$ fixed, we obtain

$$
\begin{aligned}
\frac{\partial S_{A}}{\partial l^{(2)}=} & \int_{l^{(2)}-l^{(1)}}^{\infty} d w n\left(w, l^{(2)}-\frac{w}{2}, t\right)+\int_{0}^{\infty} d w n\left(w, l^{(2)}+\frac{w}{2}, t\right) \\
& -\int_{l^{(1)}}^{l^{(2)}} d x n\left(l^{(2)}-x, \frac{x}{2}+\frac{l^{(2)}}{2}, t\right) .
\end{aligned}
$$

Finally by taking the derivative by $l^{(1)}$, we find

$$
\frac{\partial^{2} S_{A}}{\partial l^{(1)} \partial l^{(2)}}=\frac{1}{4} \frac{\partial^{2} S_{A}}{\partial \xi^{2}}-\frac{\partial^{2} S_{A}}{\partial l^{2}}=2 n\left(l^{(2)}-l^{(1)}, \frac{l^{(1)}+l^{(2)}}{2}, t\right)=2 n(l, \xi, t) .
$$




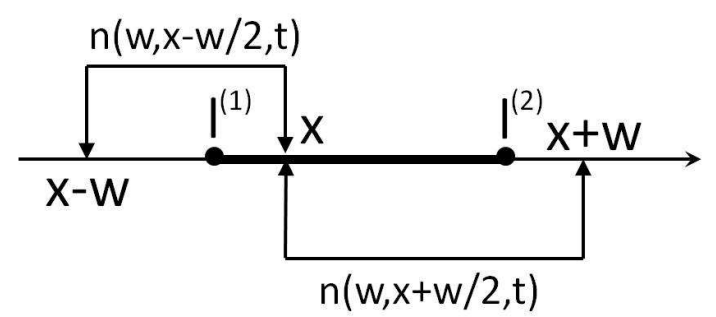

Figure 13: A sketch of expression of $S_{A}$ as a sum of entanglement density. Hooks with the arrows on both end points describe the entangling pairs between two points.

In this way, we can extract $n(l, \xi, t)$ if we know $S_{A}$ as a function of $l^{(1)}, l^{(2)}$ and $t$.

For a ground state of a $\mathrm{CFT}_{2}$ with the central charge $c, S_{A}$ is given by (4.16) and thus the entanglement density is found to be

$$
n_{C F T}(l, \xi, t)=\frac{c}{6 l^{2}} .
$$

Notice that the entanglement density is free from the UV divergences. Thus, when we talk about a deformation of a $\mathrm{CFT}_{2}$ as in the present example, it is sometimes useful to normalize the entanglement density by multiplying $l^{2}$.

\subsection{Strong Subadditivity and Positivity of Entanglement Density}

Consider a two dimensional field theory in a generically excited state. We introduce three subsystems $A_{1}, A_{2}$ and $A_{3}$ so that they are defined by the intervals $\left[l_{1}, l_{2}\right],\left[l_{2}, l_{3}\right]$ and $\left[l_{3}, l_{4}\right]$, respectively, such that $l_{1}<l_{2}<l_{3}<l_{4}$.

The strong subadditivity [33] (refer to [34] for its application to field theories and to [35] for its holographic proof) is given by the inequality

$$
S_{A_{1} \cup A_{2}}+S_{A_{2} \cup A_{3}} \geq S_{A_{1} \cup A_{2} \cup A_{3}}+S_{A_{2}} .
$$

In our setup, this is equivalent to

$$
S\left(l_{1}, l_{3}\right)+S\left(l_{2}, l_{4}\right) \geq S\left(l_{2}, l_{3}\right)+S\left(l_{1}, l_{4}\right),
$$

where $S(p, q)$ is the entanglement entropy $S_{A}$ for the interval $[p, q]$. 
In particular, we can take the limit $\delta_{1,2} \rightarrow+0$ with $l_{2}=l_{1}+\delta_{1}$ and $l_{4}=l_{3}+\delta_{2}$. Then, the strong subadditivity (5.5) is rewritten into

$$
\frac{\partial^{2} S(p, q)}{\partial p \partial q} \geq 0
$$

This proves that the entanglement entropy $n(l, \xi, t)$ in (5.3) is positive.

\subsection{Conservation Law}

It is useful to study how the total integration of the entanglement density behaves. To make the analysis simple, we assume that the $x$ coordinate can be compactified on a circle with the length $L$. The infinitely extended system we are mainly studying in this paper can be obtained in the limit $L \rightarrow \infty$. Since we have the obvious relation $n(l, \xi, t)=n(L-l, L-\xi, t)$ due to the periodicity, we can restrict the integration of $l$ to $0 \leq l \leq L / 2$. We will focus on the increased amount of entanglement entropy and entanglement density compared with the ground state, denoted by $\Delta S_{A}$ and $\Delta n$, respectively.

Thus we find

$$
\begin{aligned}
& \int_{0}^{L} d \xi \int_{0}^{L / 2} d l \Delta n(l, \xi, t)=\int_{0}^{L} d l^{(1)} \int_{l^{(1)}}^{l^{(1)}+L / 2} d l^{(2)} \Delta n(l, \xi, t) \\
& =\frac{1}{2} \int_{0}^{L} d l^{(1)} \int_{l^{(1)}}^{l^{(1)}+L / 2} d l^{(2)} \frac{\partial^{2} \Delta S_{A}}{\partial l^{(1)} \partial l^{(2)}} \\
& =\frac{1}{2} \int_{0}^{L} d l^{(1)}\left[\left.\frac{\partial}{\partial x} \Delta S_{A}\left(x, l^{(1)}+L / 2\right)\right|_{x=l^{(1)}}-\left.\frac{\partial}{\partial x} \Delta S_{A}\left(x, l^{(1)}\right)\right|_{x=l^{(1)}}\right] .
\end{aligned}
$$

We can show that the first term in the third line of (5.8) vanishes. This is because by employing the identity $S_{A}=S_{B}$ for pure states and the periodicity of the circle, we can easily show

$$
\int_{0}^{L} d y\left[\Delta S_{A}(y+\delta / 2, y+L / 2)-\Delta S_{A}(y-\delta / 2, y+L / 2)\right]=0,
$$

for any $\delta$. Moreover, the other term in the third line of (5.8) vanishes because the first law (4.23) tells us

$$
\Delta S_{A}(y+\delta / 2, y)-\Delta S_{A}(y-\delta / 2, y) \simeq \frac{\pi}{3} T_{t t} \cdot \delta^{2},
$$

in the $\delta \rightarrow 0$ limit. Remember that our first law assumes that there is a UV fixed point in the field theory we consider.

In this way, we proved that the total integration of entanglement density is constant. In other words, the total number of entangled pairs is conserved:

$$
\int_{0}^{L} d \xi \int_{0}^{L / 2} d l \Delta n(l, \xi, t)=0 .
$$


In general, the negative contribution comes from the UV region where $l$ is very small. Indeed, by using the first law (4.23), we can generally show the following result in the limit $l \rightarrow 0$ :

$$
\lim _{l \rightarrow 0} \Delta n(l, \xi, t)=-\frac{\pi}{3} T_{t t}(\xi, t)
$$

\subsection{Entanglement Density for Global Quenches}

To understand better the meaning of entanglement density, we would like to consider the global quenches in two dimensional CFTs with central charge $c$ before we examine the local quenches. As shown in [6], the increased amount of entanglement entropy for the subsystem with the width $l$ behaves like

$$
\Delta S_{A}=c \cdot \Delta m \cdot t \quad(\epsilon<t \leq l / 2), \quad \Delta S_{A}=\frac{c}{2} \cdot \Delta m \cdot l \quad(t \geq l / 2),
$$

where $\Delta m$ is the energy gap which creates the global quench times a numerical constant and is proportional to the effective temperature at late time; $\epsilon$ is cut off scal 5 of the quench process, which is of order $\delta m^{-1}$.

In this case, the entanglement density (5.3) reads

$$
\Delta n(l, \xi, t)=\frac{c \cdot \Delta m}{4} \cdot \delta(t-l / 2)+\Delta n(l, \xi, t)_{U V}
$$

where the last term denotes the UV contribution which is non-trivial only for $l$ of order $\epsilon \sim \Delta m^{-1}$ or smaller. This UV term is negative such that the conservation law (5.11) holds. The delta functional term in (5.14) shows that the entangled pairs have the range $2 t$ at time $t$. We sketched this behavior in Fig 14. This behavior can be understood that the entangled pair moves in the opposite way at the speed of light, being consistent with the interpretation in [6].

In this way, for the excited states produced by quantum quenches, $\Delta n(l, \xi, t)$ tends to positive for large $l$, while it becomes negative for small $l$ due to the conservation law and the first law as in (5.12). Therefore we learn that an excitation of a quantum system corresponds to breaking shorter range entanglement and creating longer range entanglement.

\subsection{Entanglement Density for Holographic Local Quenches}

Now let us come back to the analysis of holographic local quenches. We consider the difference from the ground state and would like to calculate $\Delta n(l, \xi, t)$ from $\Delta S_{A}(l, \xi, t)$ by using the formula (5.3). The results are plotted in Fig[15. We can confirm that the total (properly normalized) density $l^{2} n(l, \xi, t)=1+l^{2} \Delta n(l, \xi, t)$ is always positive as expected (we set $c=6$ in the plot).

\footnotetext{
${ }^{5}$ More precisely this is the length scale beyond which we can approximate the excited state by the boundary state, which preserves the boundary conformal invariance. See 14 for more details.
} 


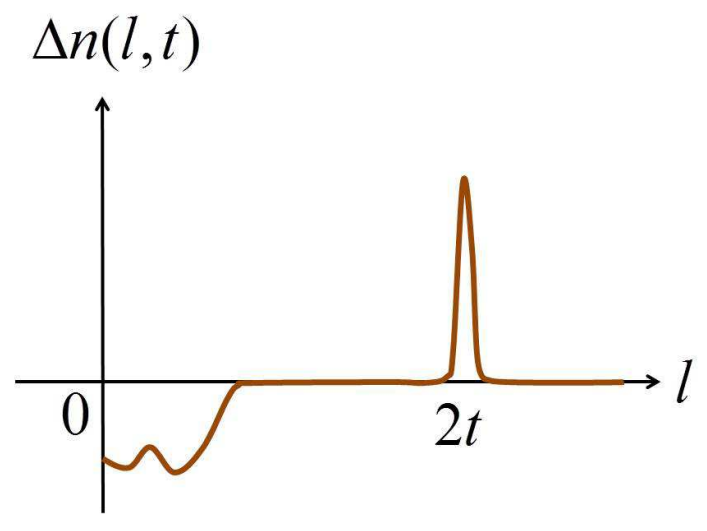

Figure 14: A sketch of the entanglement density as a function of $l$ under a global quench.

First notice that the positive peaks in Fig 15 are all delta-functional. This is because our gravity backgrounds, where we calculated the HEE, are singular due the presence of the deficit angle, corresponding to the point particle limit. This delta-functional peak appears at

$$
\xi= \pm \sqrt{l^{2} / 4-t^{2}-\alpha^{2}}
$$

which is when the massive particle is on top of $\gamma_{A}$ (see Fig 22). However, it is easy to imagine a regularization by replacing the point particle with a finite size object, which will replace a deltafunctional peak with a smooth peaks with a finite width (called $\eta$ ).

Employing this regularization 6 the entanglement density $n(l, \xi, t)=n\left(l^{(2)}-l^{(1)},\left(l^{(2)}+l^{(1)}\right) / 2, t\right)$ is plotted as a function of $l^{(1)}$ and $l^{(2)}$ in Fig 16 for $t=0$ and $t=2$. It is clear from this graph that the peaks exist along the curve (5.15) or equally $l^{(1)} \cdot l^{(2)}=-\alpha^{2}-t^{2}$ as expected from the condition that the massive particle passes through $\gamma_{A}$. Moreover, $\Delta n$ takes the largest value when $l^{(2)}=-l^{(1)}=\sqrt{\alpha^{2}+t^{2}}$.

These behaviors can be systematically understood by considering the time-evolution of each entangled pain 7 . At time $t$, the pair of the points $x=\xi-\sqrt{\xi^{2}+\alpha^{2}+t^{2}}$ and $x=\xi+\sqrt{\xi^{2}+\alpha^{2}+t^{2}}$ are entangled for any values of $\xi$. Especially the pair at $\xi=0$ (i.e. the one between $x=-\sqrt{\alpha^{2}+t^{2}}$ and $x=\sqrt{\alpha^{2}+t^{2}}$ ) possesses the strongest entanglement, which we call the dominant entangled pair. More generally the entanglement is enough strong for the entangled pairs with $|\xi| \lesssim \alpha$. Therefore they follow the time evolutions in an obvious way, as sketched in Fig.17. At earlier time

\footnotetext{
${ }^{6}$ More explicitly, we replaced the derivative $\frac{\partial^{2} S_{A}}{\partial l^{(1)} \partial l^{(2)}}$ with the second order difference $S_{A}\left(l^{(1)}+\eta / 2, l^{(2)}+\eta\right)-$ $S_{A}\left(l^{(1)}-\eta / 2, l^{(2)}+\eta\right)-S_{A}\left(l^{(1)}+\eta / 2, l^{(2)}-\eta\right)+S_{A}\left(l^{(1)}-\eta / 2, l^{(2)}-\eta\right)$.

${ }^{7}$ Another holographic realization of entangled pairs refer to [36] based on the AdS/BCFT proposal [37].
} 
$t<<\xi$ the pair moves slowly. At late time $t>>\xi$, they move at the speed of light in the opposite direction. This time evolution is also sketched with the energy density in Fig,4,

We can explain the qualitative behaviors of HEE calculated in the previous section by referring to this structure of quantum entanglement in our holographic local quenches, based on the entangled pairs. For example, let us explain the result described in Fig 12. First assume $t<l / 2$. Then if we sweep a length $l$ interval $A$ by shifting its center $\xi, S_{A}$ cannot detect the entanglement pairs generated by the local quench when $t-l / 2 \leq \xi \leq-t+l / 2$ because the dominant entangled pair is completely included in $A$ for these values of $\xi$. This missing contribution is proportional to $l / 2-t$ and therefore $S_{A}$ increases linearly until $t=l / 2$. At late time $t>l / 2$, for any $\xi$, the interval $A$ always include only one of the pair and therefore it fully contributes to $S_{A}$. Thus the integral $\int d \xi \Delta S_{A}$ no longer depends on the time $t$. These explain the behavior found in Fig 12 ,

One may think that any long range entanglement should not exist at $t=0$ because the massive particle is situated at deep UV region $z=\alpha$. However, this speculation is based on a too naive $\mathrm{UV} / \mathrm{IR}$ relation of AdS/CFT. Indeed, if we consider a two point function $\langle O(x) O(y)\rangle$ for a certain operator with a large conformal dimension, then we can evaluate this from the geodesic distance in AdS. The same geodesic distance appears in the calculation of HEE and therefore we find that a non-trivial result for two point functions can obtained when $x=0$, where the geodesic $\gamma_{A}$ passes very close to the massive particle as in right picture of Fig 2. Thus this consideration of two point functions also support the long range entanglement even at $t=0$. Another way to see this is to note that the energy density $T_{t t}$ computed in (2.24) decays only slowly as $x^{-4}$ in the long distance limit $x \rightarrow \infty$ for any non-zero $\alpha$.

In this way, we learned that the long range entanglement is non-trivial even at $t=0$ in our holographic model. In ideal models of local quenches we may not expect any long range entanglement just after the quench. However this is not a major problem because as we mentioned the dominant entangled pair is the one between $x=-\sqrt{\alpha^{2}+t^{2}}$ and $x=\sqrt{\alpha^{2}+t^{2}}$, which is short range $(\sim \alpha)$ at $t=0$.

In summary, we can understand the time evolutions of entanglement entropy $S_{A}$ in terms of those of entanglement density $n$. In our holographic local quench, the dynamical behavior of $n$ is described by the evolution of the entangled pairs as in Fig 17. At late time, the range of entanglement increases at the speed of light. As the time evolves, short range entanglement disappears and long range one is generated so that the total number of entangled pairs is conserved as in (5.11). This process looks a sort of the decoherence phenomena. 

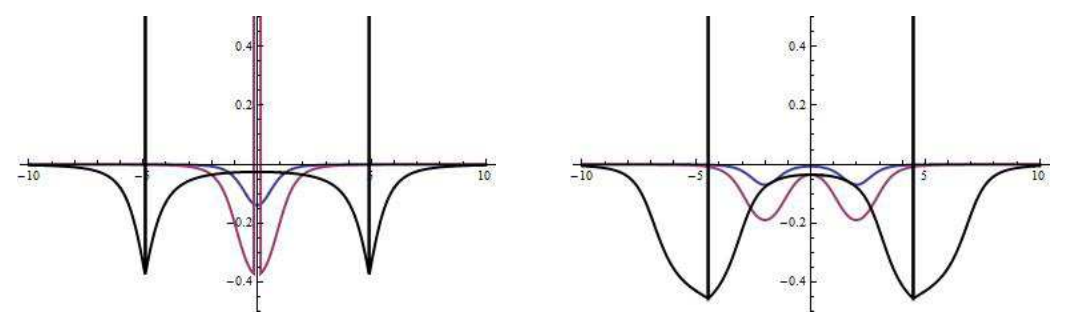

Figure 15: The numerical plots of $l^{2} \Delta n(l, \xi, t)$ as a function of the center position $\xi$ at $t=0$ (left graph) and $t=2$ (right graph). The blue, red and black graph correspond to $l=1,2,10$, respectively. We choose $M=0.5$ and set $R=\alpha=4 G_{N}=1$. Notice the relation $l^{2} n(l, \xi, t)=$ $1+l^{2} \Delta n(l, \xi, t)$
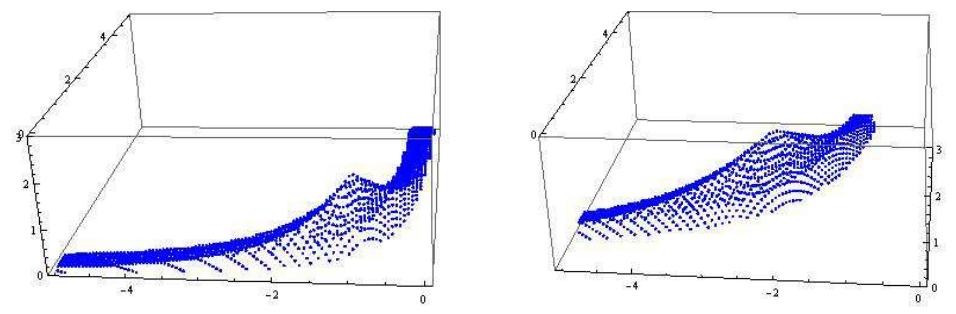

Figure 16: The numerical plots of $\Delta n(l, \xi, t)$ as a function of $l^{(1)}=\xi-l / 2<0$ (horizontal axis) and $l^{(2)}=\xi+l / 2>0$ (inward axis). We choose the regularization parameter as $\eta=0.4$. The left and right plot corresponds to $t=0$ and $t=2$, respectively. We only plot those points where $\Delta n(l, \xi, t)$ is positive. We choose $M=0.5$ and set $R=\alpha=4 G_{N}=1$.

\section{Quantum Information, Thermodynamics and Gravity}

In this section we employ the results found in the previous sections to develop some insights on connections between the quantum information and gravity via the AdS/CFT correspondence.

\subsection{Total Amount of Quantum Information}

In the CFT side, the entanglement entropy $S_{A}$ is interpreted as the entropy for an observer in $A$, assuming that the observer is not accessible to the region $B$. In order words this measures the amount of quantum information inside $B$ for such an observer. Therefore $\Delta S_{A}$ can be regarded as the amount of quantum information in $B$ which is induced by the local quench, such as the data of excited particles etc.

Notice that the standard thermal entropy for a mixed state, which is dual to a black hole entropy via $\mathrm{AdS} / \mathrm{CFT}$, corresponds to the information which is not accessible to the observer by any means 


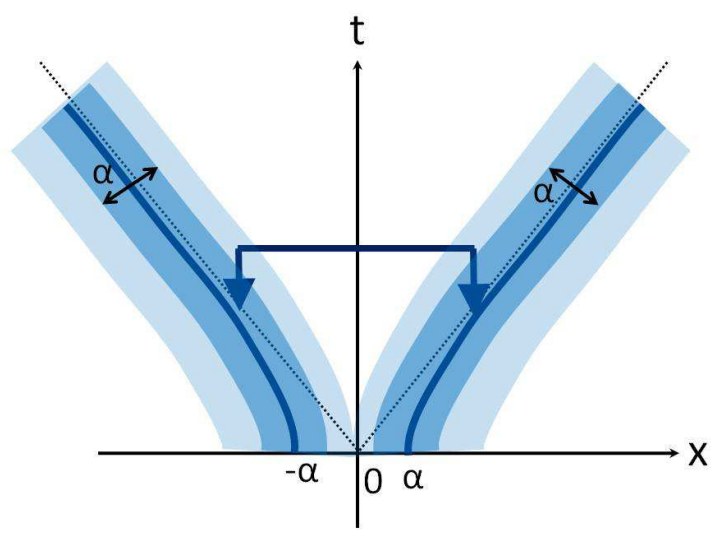

Figure 17: A sketch of time evolution of entangling pairs. The blue thick curve describes the dominant entangled pair $\xi=0$. Around this, other entangled pairs are gathered with the width of order $\alpha$.

(except for making microscopic observations of its heat bath). On the other hand, the entanglement entropy $S_{A}$ measures the amount of information which is possessed by $B$ and is accessible to the observe in $A$ after all possible experiments are done.

Therefore, the holographic entanglement entropy allows us to calculate such a novel quantity, i.e. the amount of information $\Delta S_{A}$, for any massive object in AdS, which is dual to a certain excited state in the CFT. Apply this idea to our falling particle in AdS. The standard principle in AdS/CFT tells us that the size $l$ of the localized excitations induced by the local quench is estimated as $l \sim \sqrt{t^{2}+\alpha^{2}}$, which is the value of the coordinate $z$ of the falling particle. Therefore it is natural that we choose the subsystem $A$ (or equally 8 ) to be the ball with the radius $\sqrt{t^{2}+\alpha^{2}}$. Indeed, we can see from our perturbative results (3.7) and (3.11) that $\Delta S_{A}$ takes the maximal value when $l=\sqrt{t^{2}+\alpha^{2}}$ as a function of $l$ and takes a time-independent constant value. Therefore we call it $\Delta S^{\max }$. This is evaluated as the following simple form for any dimension $d$ :

$$
\Delta S^{\max }=C_{s} \cdot m R=C_{s} \cdot \Delta
$$

where $C_{s}$ is an order one constant. Explicitly, in each dimension $d$, we find $C_{s}=2(d=2)$, $C_{s}=\pi / 2(d=3)$ and $C_{S}=4(d=4)$. We used the well-known relation between the mass $m$ of a particle and the conformal dimension $\Delta$ of its dual operator [10] assuming $\Delta>>1$. For example,

\footnotetext{
${ }^{8}$ Notice that since we are considering a pure state we always have $S_{A}=S_{B}$ and therefore the distinction between $A$ and $B$ is not important.
} 
in the $\mathrm{AdS}_{3}$ case the exact calculation (4.8) leads to at $l=\sqrt{t^{2}+\alpha^{2}}$ :

$$
\Delta S^{\max }=\frac{c}{3} \log \left(\frac{R}{\sqrt{R^{2}-M}} \sin \left(\frac{\pi \sqrt{R^{2}-M}}{2 R}\right)\right) .
$$

This shows that $\Delta S^{\max }$ is a constant which is independent from $l$. Assuming the range $1<<\Delta<<$ $c$ we finds

$$
\Delta S^{\max } \simeq 2 \Delta
$$

In this way we find the number of miscrostates of the massive particle is estimated as follows in any $d$ :

$$
\text { \# Microstates } \sim e^{\Delta S^{\max }}=e^{C_{s} \cdot \Delta} \text {. }
$$

This is qualitatively consistent with the well-known Hagedron growth of degeneracy of the states with a large conformal dimension $\Delta>>1$ [25, 38]. Remember that this relation (6.4) holds only when the backreaction of the massive particle is small. For example, this requires $\Delta<<c$ in $d=2$ as can be seen from (6.2).

If we remember that the energy $E$ of localized excitations made of radiations in the CFT is given by (2.5), then we find the simple relation between the amount of information of this excited lump and its the total energy:

$$
\Delta S^{\max } \sim E \cdot \alpha
$$

Remember that $\alpha$ denotes the size of the lump at the initial time $t=0$. This provides us a prediction that in the large $N$ strong coupled gauge theories, the amount of information possessed by such a radiation 'fire ball' is given by its energy $E$ times its linear size $\alpha$. It would be very intriguing future problem to extend this estimation to other objects like the fundamental strings or D-branes in AdS/CFT in order to see how much the relation (6.5) is universal.

Finally, we would also like to comment on a possible interpretation of $\Delta S^{\max }$ from the gravity side. Since the Unruh temperature of the falling massive particle reads $T_{U}=\frac{1}{2 \pi \alpha}$ at $t=0$, we can rewrite this relation (6.5) into

$$
T_{U} \cdot \Delta S^{\max } \sim E
$$

which looks like the thermodynamical first law. We can speculate that this is the thermodynamical relation which is understood as that for a Rindler observer, whose acceleration is $\alpha^{-1}$. This interpretation makes sense near $t=0$ where the energy from the viewpoint of the Rindler observer coincides with $E$.

\footnotetext{
${ }^{9}$ If we assume a very large dim. operator $\Delta>c$ in the local thermal quench, then it is dual to a BTZ black hole. In this case we find from (6.2) $\Delta S^{\max } \simeq \frac{\pi \sqrt{M}}{4 G_{N}}=\frac{\pi}{3} \sqrt{3 c \Delta}$. This is a half of the black hole entropy and agrees with the Cardy formula.
} 


\subsection{Entanglement Renormalization and Origin of Gravitational Force}

A helpful framework to study the connection between quantum entanglement of a given quantum many-body system and its holographic geometry is the entanglement renormalization (or called multi-scale entanglement renormalization ansatz, MERA) [39]. Indeed, it has been conjectured in [40] that the geometry described by a tensor network of a quantum critical system in MERA describes the AdS spacetime dual to this critical point. In [41, this correspondence has been studied in the continuum limit (i.e. field theory limit), called cMERA [42] and a candidate of holographic metric in terms of purely field theoretic data has been proposed. Refer also to [43] for recent developments in this subject.

Motivated by this it is intriguing to see how we can describe the time evolution of local quenches from the viewpoint of the entanglement renormalization. A general framework to describe a quantum state graphically is called tensor networks (see e.g. reviews [44]) and has been recently used to prepare a candidate of approximate ground states based on the variational principle, optimized by a number of parameters.

The tensor network for a CFT is called MERA and the network for its ground state is described in Fig 18, It describes the coarse-graining procedure of a given quantum system such as spin chains as we goes from the bottom (UV) to the top (IR), by combining two sites (or spins) into one, which looks like a tree in Fig 18. To realize the entanglement structure of CFTs, we need to add so called disentanglers which are unitary transformations between two spins and which are written as the horizontal ladders in Fig 18 ,

Let us define the number of disentanglers such that each of them carries the entanglement entropy $\log 2$. Then we find that the number of disentanglers in each bond of the MERA network is given by $\frac{c}{6}$. Indeed, this reproduces the correct entanglement entropy for ground states in CFTs given by the well-known formula (4.10) as explained in Fig.18. Notice that we can estimate the entanglement entropy from the MERA diagram by encircling the subsystem $A$ and counting the number of intersecting bonds (disentanglers). Though there are many choices of encircling curve, we can optimize the result by choosing the one with minimal number of intersecting bonds. This minimizing procedure nicely matches with the HEE formula (3.1) as pointed out in [40].

By comparing this estimation of the entanglement entropy in CFTs with (5.4), we can speculate that the number of disentanglers in a bond is roughly approximated by $l^{2} \cdot \overline{n(l, \xi, t)}$ in more general tensor networks which do not correspond to ground states of CFTs. Here the averaging symbol means an average like $\sim \frac{1}{l^{2}} \int_{0}^{l} d l \int_{\xi-l / 2}^{\xi+l / 2} d \xi$. The conservation law (5.11) tells us that the total number of disentangles is also conserved. Moreover, we can roughly speculate the relation between the entanglement density and the holographic metric in the extra direction $z: \sqrt{g_{z z}(z, x, t)} \propto l \cdot \overline{n(l, \xi, t)}$ 


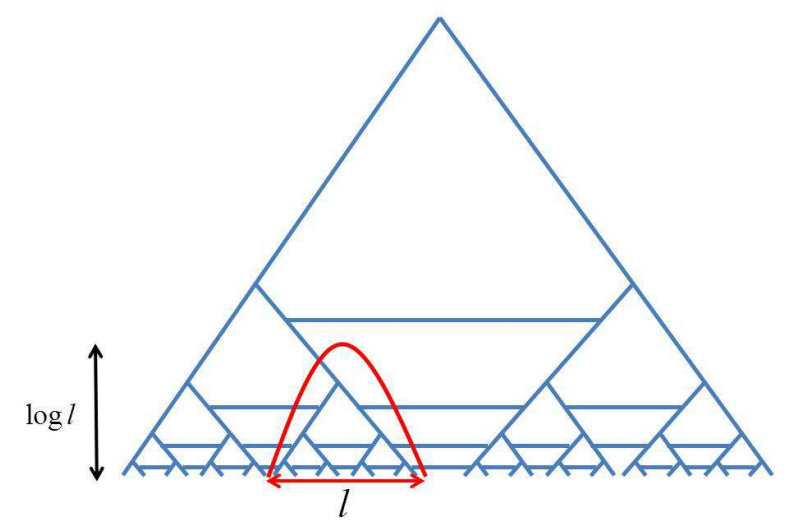

Figure 18: The tensor network of MERA for a ground state of a CFT. The coarse-graining procedure is done from the bottom (UV) to the top (IR). The horizontal ladders represent the disentanglers, while the tree structure does the coarse-graining procedure, combining two sites into one. We also show the estimation of entanglement entropy when the subsystem $A$ is an interval with length $l$ by counting the number of bonds.

with the identification $l=z$ and $\xi=x$. It will be an interesting future problem to work out these relations more precisely.

Now we would like to consider how to describe local quenches by using tensor networks. We consider both our holographic local quench and the original local quench in 8 . We argue that they are described by the left and right pictures of Fig[19, respectively. In our model, there are disentanglers even in IR regions because we start with the ground state of a CFT on an infinite line. On the other hand, in the local quench induced by the joining two semi-infinite lines, there should be no entanglement between the two in the IR region. As in (4.10) and (4.16), we can confirm that the behavior of $S_{A}$ when $t>>l$ is the same as that for the ground state. Thus the MERA structures in the UV region are also the same as that for the ground state in both cases. Finally, the massive falling particle corresponds to the insertion of many disentanglers. This description is consistent with the behaviors (4.20) and (4.21) at late time.

Finally we would like to ask what is the holographic origin of gravitational force. As we have seen, the propagation of entangled pairs is dual to the falling particle in AdS. This tells us that the evolution of the range of entanglement is dual to the gravitational force in AdS space. Therefore we can argue that the gravitational force is dual to a kind of decoherence which breaks the short range entanglement and tries to expand the range of entanglement. In MERA, this is described by the motion of the dense lump of disentanglers toward upper direction as depicted in Fig 19 , 

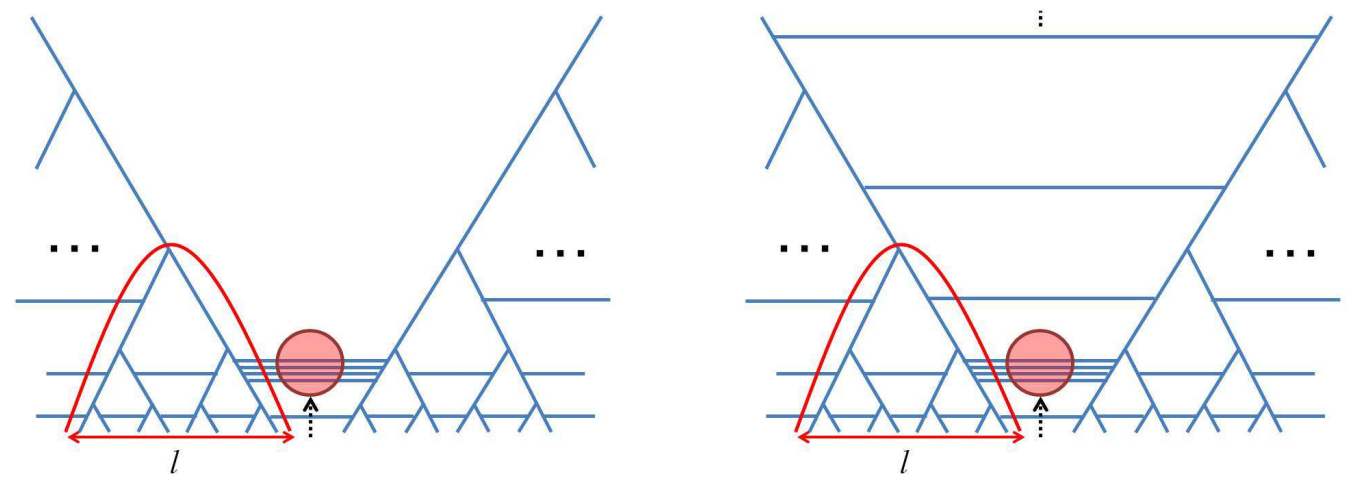

Figure 19: The tensor networks of MERA after local quenches in a CFT. The left picture corresponds to the local quench triggered by joining two CFTs on semi-infinite lines. The right one describes our holographic local quench induced by local excitations in the CFT.

\section{Conclusions and Discussions}

In the first half of this paper, we proposed a holographic model of local quench and study its property especially focusing on the time evolution of entanglement entropy. In our model, the local quench is simply described by a free falling massive particle in an AdS space. We performed a perturbative analysis of its back-reacted geometry and computed the holographic entanglement entropy (HEE) in any dimension as well as the holographic energy stress tensor. Moreover, we presented exact calculations of HEE for local quenches in two dimensions. We leave exact calculations in higher dimensional cases for a future problem. Since in our model we considered a massive particle without any charge, it will be another interesting future problem to extend our results to charged massive particles.

In most of earlier works on local quenches, local excitations are generated by joining two semiinfinite lines (or spin chains). On the other hands, our holographic model corresponds to the setup where local excitations are induced in a CFT on an infinite line and does not involve any joining procedure. Therefore we found that details of the time-evolution of entanglement entropy are quantitatively different between them, while we find that the qualitative behavior agrees with each other. For example, the logarithmic time evolution $\sim \log t$ is common to both of them for a large subsystem. However, its coefficients differ by the factor two between them and we give an simple explanation from the viewpoint of entanglement renormalization. This is because there exists long range entanglement even just after the quench. Even though we were able to reproduce the basic properties of local quenches in our model, it is an intriguing future problem to construct a clean gravity dual without any long range entanglement. 
In order to study the time evolution of quantum entanglement more clearly, we introduced a new quantity called the entanglement density for two dimensional field theories. This measures the density of entangled pairs between given two points. We showed that the strong subadditivity guarantees that this quantity is positive as should be so if we want to understand it as a physical density. Moreover, we found a simple relation between the entanglement density and the energy density in the small subsystem limit.

It will be interesting to generalize this quantity so that we can incorporate the effect of threebody or higher entanglement. At least it is already clear that $N$-body entanglement is related to a $N$-th derivative of the entanglement entropy for a suitable subsystem. At the same time, it will be an important problem to extend the entanglement density into higher dimensional counterparts and study various holographic models. A special example of higher dimensional entanglement density is obtained by replacing the interval subsystems with strip ones.

In the final part of this paper, we calculated the amount of quantum information carried by a massive object (localized excitations) in the dual CFT. We find a rather simple rule that this amount of information is given by the energy $E$ of the object times it linear size $\alpha$. This is expected to be a prediction which is true for large $N$ strongly coupled gauge theories. It will very intriguing to see how this behavior is universal in more general cases.

\section{Acknowledgements}

TT would like to thank E. Tonni for earlier stimulating collaborations on local quenches and valuable remarks on the draft of this paper. We are also grateful to M. Fujita, M. Hotta, V. Hubeny, L-Y. Hung, H. Matsueda, M. Rangamani and T. Ugajin for useful discussions, and especially to R. Myers for reading the draft of this paper and giving us useful comments. MN would like to thank the Perimeter Institute for giving the opportunities to discuss with researchers. TT is supported by JSPS Grant-in-Aid for Challenging Exploratory Research No.24654057. TT is also supported by World Premier International Research Center Initiative (WPI Initiative) from the Japan Ministry of Education, Culture, Sports, Science and Technology (MEXT).

\section{A Perturbative Construction of Gravity Duals}

In this appendix, we will show a calculation of the back-reaction for a falling massive particle in $\mathrm{AdS}_{4}$ by solving (2.7) and (2.8) directly. 


\section{A.1 Perturbation Theory}

We focus on $d=3$ i.e. the $\mathrm{AdS}_{4}$ case and define $x=x_{1}$ and $y=x_{2}$. We will set $R=1$ just for simplicity. We perform the Fourier transformation with respect to $x, y$ and $t$. Using the symmetry $x \rightarrow-x$ and $y \rightarrow-y$, we can write down the following ansatz of the perturbed metric:

$$
\begin{aligned}
& \delta g_{t t}=h(z) e^{i\left(k_{x} \cdot x+k_{y} \cdot y-\omega t\right)}, \quad \delta g_{t x}=\frac{k_{x}}{\omega} \cdot w(z) e^{i\left(k_{x} \cdot x+k_{y} \cdot y-\omega t\right)}, \quad \delta g_{t y}=\frac{k_{y}}{\omega} \cdot w(z) e^{i\left(k_{x} \cdot x+k_{y} \cdot y-\omega t\right)}, \\
& \delta g_{x x}=\left(k_{x}^{2} f(z)+g(z)\right) e^{i\left(k_{x} \cdot x+k_{y} \cdot y-\omega t\right)}, \quad \delta g_{x y}=k_{x} k_{y} f(z) e^{i\left(k_{x} \cdot x+k_{y} \cdot y-\omega t\right)}, \\
& \delta g_{y y}=\left(k_{y}^{2} f(z)+g(z)\right) e^{i\left(k_{x} \cdot x+k_{y} \cdot y-\omega t\right)} .
\end{aligned}
$$

The energy stress tensor for the falling particle described by $z(t)=\sqrt{t^{2}+\alpha^{2}}$ after the Fourier transformation

$$
T^{\mu \nu}\left(k_{x}, k_{y}, \omega, z\right)=\frac{1}{(2 \pi)^{3}} \int d x d y d t e^{-i\left(k_{x} \cdot x+k_{y} \cdot y-\omega t\right)} \cdot T^{\mu \nu}(x, y, t, z) .
$$

is given by

$$
\begin{aligned}
& T^{z z}=2 \hat{m} \cdot \frac{z^{5} \sqrt{z^{2}-\alpha^{2}}}{\alpha} \cdot \cos \left(\omega \sqrt{z^{2}-\alpha^{2}}\right), \\
& T^{z t}=2 i \hat{m} \cdot \frac{z^{6}}{\alpha} \cdot \sin \left(\omega \sqrt{z^{2}-\alpha^{2}}\right), \\
& T^{t t}=2 \hat{m} \cdot \frac{z^{7}}{\alpha \sqrt{z^{2}-\alpha^{2}}} \cdot \cos \left(\omega \sqrt{z^{2}-\alpha^{2}}\right),
\end{aligned}
$$

and other components are vanishing. We employed the identity

$$
\delta\left(z-\sqrt{t^{2}+\alpha^{2}}\right)=\frac{z}{\sqrt{z^{2}-\alpha^{2}}}\left(\delta\left(t-\sqrt{z^{2}-\alpha^{2}}\right)+\delta\left(t+\sqrt{z^{2}-\alpha^{2}}\right)\right),
$$

where we assumed $z \geq 0$. We also defined 'normalized mass'

$$
\hat{m} \equiv \frac{8 \pi G_{N} m}{(2 \pi)^{3}} .
$$

It is straightforward to check that they satisfy the conservation law $\nabla_{\mu} T^{\mu \nu}=0$.

\section{A.2 General Solutions to Einstein Equation}

By evaluating the left-hand side of (2.7), in the end we find that all the solutions can be constructed as follows: First we define $u(z)$ such that

$$
\begin{aligned}
& w(z)=A(1-\omega)+\frac{\omega^{2}}{2} u(z)-\frac{u^{\prime}(z)}{z}+\frac{u^{\prime \prime}(z)}{2}, \\
& f(z)=-\frac{A}{\omega}-\frac{u(z)}{2}-\frac{u^{\prime}(z)}{z \omega^{2}}+\frac{u^{\prime \prime}(z)}{2 \omega^{2}}, \\
& g(z)=\frac{u^{\prime}(z)}{z}+\frac{4 u^{\prime}(z)}{z^{3} \omega^{2}}-\frac{u^{\prime \prime}(z)}{2}-\frac{4 u^{\prime \prime}(z)}{z^{2} \omega^{2}}+\frac{2 u^{(3)}(z)}{z \omega^{2}}-\frac{u^{(4)}(z)}{2 \omega^{2}}, \\
& h(z)=A \omega-\frac{\omega^{2}}{2} u(z)+\frac{2 u^{\prime}(z)}{z}+\frac{4 u^{\prime}(z)}{\omega^{2} z^{3}}-u^{\prime \prime}(z)-\frac{4 u^{\prime \prime}(z)}{\omega^{2} z^{2}}+\frac{2 u^{(3)}}{z \omega^{2}}-\frac{u^{(4)}}{2 \omega^{2}} .
\end{aligned}
$$


Moreover, we define $y(z)$ by

$$
y(z)=\frac{u^{\prime}(z)}{z} .
$$

Then we can show that (A.6) is a solution to (2.7) if the following equation is satisfied

$$
y^{(3)}(z)-\left(k_{x}^{2}+k_{y}^{2}-\omega^{2}\right) y^{\prime}(z)-\frac{4 \hat{m}}{\omega}\left[\sin \left(\omega \sqrt{z^{2}-\alpha^{2}}\right)-\omega \sqrt{z^{2}-\alpha^{2}} \cos \left(\omega \sqrt{z^{2}-\alpha^{2}}\right)\right]=0 .
$$

It is clear that there are five integration constants. They are dual to the background metric perturbation (four parameters) and the boundary energy stress tensor (one parameter).

\section{A.3 Special Solutions: $\alpha \rightarrow 0$}

We can solve (A.8) in the particular case $\alpha \rightarrow 0$ where $z(t) \simeq|t|$. We impose the boundary conditions such that there are no non-normalize deformations (i.e. the background metric is not perturbed) and that the metric perturbation does not blow up in the IR limit $z \rightarrow \infty$. This uniquely fixed the form of $u(z)$ as follows

$$
\begin{aligned}
u(z)= & -\frac{4 \hat{m}}{\alpha \omega^{4}\left(k_{x}^{2}+k_{y}^{2}-\omega^{2}\right)^{2}}\left(4 k_{x}^{2}+4 k_{y}^{2}-6 \omega^{2}+z^{2} \omega^{2}\left(k_{x}^{2}+k_{y}^{2}-\omega^{2}\right)\right. \\
& +\frac{\left(k_{x}^{2}+k_{y}^{2}-\omega^{2}\right)^{2}}{\left(k_{x}^{2}+k_{y}^{2}\right)^{2}}\left(\left(-4 k_{x}^{2}-4 k_{y}^{2}-2 \omega^{2}+\left(k_{x}^{2}+k_{y}^{2}\right) z^{2} \omega^{2}\right) \cos (z \omega)-2 z \omega\left(2 k_{x}^{2}+2 k_{y}^{2}+\omega^{2}\right) \sin (z \omega)\right) \\
& \left.+\frac{1}{\left(k_{x}^{2}+k_{y}^{2}\right)^{2}} \cdot 2 e^{-z \sqrt{k_{x}^{2}+k_{y}^{2}-\omega^{2}}} \omega^{6}\left(1+z \sqrt{k_{x}^{2}+k_{y}^{2}-\omega^{2}}\right)\right) .
\end{aligned}
$$

We also have the condition $A=0$. These completely fix $f(z), g(z), h(z)$ and $w(z)$.

Near the AdS boundary $z \rightarrow 0$ they behave

$$
\begin{aligned}
& w(z) \simeq-\frac{4 \hat{m} \omega^{2}}{3 \alpha\left(k_{x}^{2}+k_{y}^{2}\right) \sqrt{k_{x}^{2}+k_{y}^{2}-\omega^{2}}} \cdot z^{3}, \\
& f(z) \simeq-\frac{4 \hat{m}\left(k_{x}^{2}+k_{y}^{2}-2 \omega^{2}\right)}{3 \alpha\left(k_{x}^{2}+k_{y}^{2}\right)^{2} \sqrt{k_{x}^{2}+k_{y}^{2}-\omega^{2}}} \cdot z^{3}, \\
& g(z) \simeq \frac{4 \hat{m}\left(k_{x}^{2}+k_{y}^{2}-\omega^{2}\right)}{3 \alpha\left(k_{x}^{2}+k_{y}^{2}\right) \sqrt{k_{x}^{2}+k_{y}^{2}-\omega^{2}}} \cdot z^{3}, \\
& h(z) \simeq \frac{4 \hat{m}}{3 \sqrt{k_{x}^{2}+k_{y}^{2}-\omega^{2}}} \cdot z^{3} .
\end{aligned}
$$


We can read off the tensor $t_{a b}$ defined by (2.17) as follows:

$$
\begin{aligned}
& t_{t t}=\frac{4 \hat{m}}{3 \alpha \sqrt{k_{x}^{2}+k_{y}^{2}-\omega^{2}}}, \\
& t_{t x}=-\frac{4 \hat{m} k_{x} \omega}{3 \alpha\left(k_{x}^{2}+k_{y}^{2}\right) \sqrt{k_{x}^{2}+k_{y}^{2}-\omega^{2}}}, \\
& t_{t y}=-\frac{4 \hat{m} k_{y} \omega}{3 \alpha\left(k_{x}^{2}+k_{y}^{2}\right) \sqrt{k_{x}^{2}+k_{y}^{2}-\omega^{2}}}, \\
& t_{x x}=\frac{4 \hat{m}\left(k_{y}^{4}+k_{x}^{2} k_{y}^{2}-k_{y}^{2} \omega^{2}+\omega^{2} k_{x}^{2}\right)}{3 \alpha\left(k_{x}^{2}+k_{y}^{2}\right)^{2} \sqrt{k_{x}^{2}+k_{y}^{2}-\omega^{2}}}, \\
& t_{x y}=-\frac{4 \hat{m} k_{x} k_{y}\left(k_{x}^{2}+k_{y}^{2}-2 \omega^{2}\right)}{3 \alpha\left(k_{x}^{2}+k_{y}^{2}\right)^{2} \sqrt{k_{x}^{2}+k_{y}^{2}-\omega^{2}}}, \\
& t_{y y}=\frac{4 \hat{m}\left(k_{x}^{4}+k_{x}^{2} k_{y}^{2}-k_{x}^{2} \omega^{2}+\omega^{2} k_{y}^{2}\right)}{3 \alpha\left(k_{x}^{2}+k_{y}^{2}\right)^{2} \sqrt{k_{x}^{2}+k_{y}^{2}-\omega^{2}}} .
\end{aligned}
$$

The physical holographic stress tensor $T_{a b}$ is calculated from (2.17) via the formula (2.18). In the end we will find that (A.11) reproduces the result (2.20) at $\alpha=0$. For example, if we take the $\alpha \rightarrow 0$ limit of (2.20), we can show

$$
T_{t t}(t, \rho) \rightarrow \frac{M}{2 \pi G_{N} R \alpha} \delta\left(\rho^{2}-t^{2}\right)
$$

by using

$$
\lim _{\alpha \rightarrow 0} \frac{\alpha^{4}}{\left(x^{2}+\alpha^{2} r^{2}\right)^{\frac{5}{2}}}=\frac{4}{3 r^{4}} \delta(x) .
$$

By performing the Fourier transformation, we find

$$
T_{t t}\left(\omega, k_{x}, k_{y}\right) \rightarrow \frac{M}{8 \pi^{3} G_{N} R \alpha}\left(k_{x}^{2}+k_{y}^{2}-\omega^{2}\right)^{-1 / 2} .
$$

This precisely agrees with (A.11).

\section{References}

[1] J. Eisert, M. Cramer and M. B. Plenio, "Area laws for the entanglement entropy - a review," Rev. Mod. Phys. 82 (2010) 277 [arXiv:0808.3773 [quant-ph]].

[2] P. Calabrese and J. Cardy, "Entanglement entropy and conformal field theory," J. Phys. A A 42 (2009) 504005 arXiv:0905.4013 [cond-mat.stat-mech]].

[3] H. Casini and M. Huerta, "Entanglement entropy in free quantum field theory," J. Phys. A A 42 (2009) 504007 arXiv:0905.2562 [hep-th]].

[4] J. I. Latorre, A. Riera, "A short review on entanglement in quantum spin systems," J. Phys. A 42 (2009) 504002 arXiv:0906.1499 [hep-th]]. 
[5] T. Nishioka, S. Ryu and T. Takayanagi, "Holographic Entanglement Entropy: An Overview," J. Phys. A 42 (2009) 504008; T. Takayanagi, "Entanglement Entropy from a Holographic Viewpoint," Class. Quant. Grav. 29 (2012) 153001 [arXiv:1204.2450 [gr-qc]].

[6] P. Calabrese and J. L. Cardy, "Evolution of Entanglement Entropy in One-Dimensional Systems," J. Stat. Mech. 04 (2005) P04010, cond-mat/0503393 P. Calabrese and J. L. Cardy, "Time-dependence of correlation functions following a quantum quench," Phys.Rev.Lett.96(2006)136801; P. Calabrese and J. L. Cardy, "Quantum Quenches in Extended Systems," J. Stat. Mech. 06 (2007) P06008, arXiv:0704.1880; S. Sotiriadis and J. Cardy, "Quantum quench in interacting field theory: A Self-consistent approximation," Phys. Rev. B 81 (2010) 134305 arXiv:1002.0167] [quant-ph]].

[7] V. Eisler, I. Peschel, "Evolution of entanglement after a local quench," J. Stat. Mech. (2007) P06005, cond-mat/0703379;

V. Eisler, D. Karevski, T. Platini, I. Peschel, "Entanglement evolution after connecting finite to infinite quantum chains," J. Stat. Mech. (2008) P01023, arXiv:0711.0289.

[8] P. Calabrese and J. L. Cardy, "Entanglement and correlation functions following a local quench: a conformal field theory approach," J. Stat. Mech. 10 (2007) P10004, arXiv:0708.3750.

[9] J. M. Maldacena, "The large N limit of superconformal field theories and supergravity," Adv. Theor. Math. Phys. 2 (1998) 231 [Int. J. Theor. Phys. 38 (1999) 1113] arXiv:hep-th/9711200];

[10] S. S. Gubser, I. R. Klebanov and A. M. Polyakov, "Gauge theory correlators from non-critical string theory," Phys. Lett. B 428, 105 (1998); E. Witten, "Anti-de Sitter space and holography," Adv. Theor. Math. Phys. 2, 253 (1998).

[11] O. Aharony, S. S. Gubser, J. M. Maldacena, H. Ooguri and Y. Oz, "Large N field theories, string theory and gravity," Phys. Rept. 323 (2000) 183 hep-th/9905111.

[12] S. Bhattacharyya and S. Minwalla, "Weak Field Black Hole Formation in Asymptotically AdS Spacetimes," JHEP 0909 (2009) 034 [arXiv:0904.0464 [hep-th]]; S. R. Das, T. Nishioka and T. Takayanagi, "Probe Branes, Time-dependent Couplings and Thermalization in AdS/CFT," JHEP 1007 (2010) 071 arXiv:1005.3348 [hep-th]]; H. Ebrahim and M. Headrick, "Instantaneous Thermalization in Holographic Plasmas," arXiv:1010.5443 [hep-th]; D. Garfinkle and L. A. Pando Zayas, "Rapid Thermalization in Field Theory from Gravitational Collapse," Phys. Rev. D 84 (2011) 066006 [arXiv:1106.2339 [hep-th]]; P. Basu and S. R. Das, "Quantum Quench across a Holographic Critical Point," JHEP 1201 (2012) 103 arXiv:1109.3909 [hep-th]]; S. R. Das, "Holographic Quantum Quench," J. Phys. Conf. Ser. 343 (2012) 012027 [arXiv:1111.7275 [hep-th]]; E. Caceres and A. Kundu, "Holographic Thermalization with Chemical Potential," JHEP 1209 (2012) 055 arXiv:1205.2354 [hep-th]]; A. Buchel, L. Lehner and R. C. Myers, "Thermal quenches in $\mathrm{N}=2^{*}$ plasmas," JHEP 1208 (2012) 049 arXiv:1206.6785 [hep-th]]; M. J. Bhaseen, J. P. Gauntlett, B. D. Simons, J. Sonner and T. Wiseman, "Holographic Superfluids and the Dynamics of Symmetry Breaking," arXiv:1207.4194 [hep-th]. P. Basu, D. Das, S. R. Das and T. Nishioka, "Quantum Quench Across a Zero Temperature Holographic Superfluid Transition," arXiv:1211.7076] [hepth]. X. Gao, A. M. Garcia-Garcia, H. B. Zeng and H. -Q. Zhang, "Lack of thermalization in holographic superconductivity," arXiv:1212.1049 [hep-th]. W. H. Baron, D. Galante and M. Schvellinger, "Dynamics of holographic thermalization," arXiv:1212.5234 [hep-th]; A. Buchel, L. Lehner, R. C. Myers and A. van Niekerk, "Quantum quenches of holographic plasmas," arXiv:1302.2924 [hep-th].

[13] J. Abajo-Arrastia, J. Aparicio and E. Lopez, "Holographic Evolution of Entanglement Entropy," JHEP 1011 (2010) 149 [arXiv:1006.4090 [hep-th]]; T. Albash and C. V. Johnson, "Evolution of Holographic Entanglement Entropy after Thermal and Electromagnetic Quenches," New J. Phys. 13 (2011) 045017 arXiv:1008.3027 [hep-th]]; V. Keranen, E. Keski-Vakkuri and L. Thorlacius, "Thermalization and entanglement following a non-relativistic holographic 
quench," Phys. Rev. D 85 (2012) 026005 arXiv:1110.5035 [hep-th]]; D. Galante and M. Schvellinger, "Thermalization with a chemical potential from AdS spaces," JHEP 1207 (2012) 096 arXiv:1205.1548 [hep-th]]; A. Bernamonti, N. Copland, B. Craps and F. Galli, "Holographic thermalization of mutual and tripartite information in 2d CFTs," arXiv:1212.0848 [hep-th].

[14] T. Takayanagi and T. Ugajin, "Measuring Black Hole Formations by Entanglement Entropy via Coarse-Graining," JHEP 1011 (2010) 054 [arXiv:1008.3439 [hep-th]].

[15] M. M. Roberts, "Time evolution of entanglement entropy from a pulse," arXiv:1204.1982 [hep-th].

[16] S. Ryu and T. Takayanagi, "Holographic derivation of entanglement entropy from AdS/CFT," Phys. Rev. Lett. 96 (2006) 181602; "Aspects of holographic entanglement entropy," JHEP 0608 (2006) 045.

[17] V. E. Hubeny, M. Rangamani and T. Takayanagi, "A Covariant holographic entanglement entropy proposal," JHEP 0707 (2007) 062 [arXiv:0705.0016 [hep-th]].

[18] V. Balasubramanian, A. Bernamonti, J. de Boer, N. Copland, B. Craps, E. Keski-Vakkuri, B. Muller and A. Schafer et al., "Thermalization of Strongly Coupled Field Theories," Phys. Rev. Lett. 106 (2011) 191601 [arXiv:1012.4753 [hep-th]]. "Holographic Thermalization," Phys. Rev. D 84 (2011) 026010 [arXiv:1103.2683 [hep-th]].

[19] A. Allais and E. Tonni, "Holographic evolution of the mutual information," JHEP 1201 (2012) 102 arXiv:1110.1607 [hep-th]]; R. Callan, J. -Y. He and M. Headrick, "Strong subadditivity and the covariant holographic entanglement entropy formula," JHEP 1206 (2012) 081 arXiv:1204.2309 [hep-th]]. A. C. Wall, "Maximin Surfaces, and the Strong Subadditivity of the Covariant Holographic Entanglement Entropy," arXiv:1211.3494 [hep-th].

[20] K. Narayan, T. Takayanagi and S. P. Trivedi, "AdS plane waves and entanglement entropy," arXiv:1212.4328 [hep-th].

[21] G. T. Horowitz and N. Itzhaki, "Black holes, shock waves, and causality in the AdS / CFT correspondence," JHEP 9902 (1999) 010 hep-th/9901012].

[22] U. H. Danielsson, E. Keski-Vakkuri and M. Kruczenski, "Vacua, propagators, and holographic probes in AdS / CFT," JHEP 9901 (1999) 002 (hep-th/9812007.

[23] J. J. Friess, S. S. Gubser, G. Michalogiorgakis and S. S. Pufu, "Expanding plasmas and quasinormal modes of anti-de Sitter black holes," JHEP 0704 (2007) 080 [hep-th/0611005].

[24] P. Figueras, V. E. Hubeny, M. Rangamani and S. F. Ross, "Dynamical black holes and expanding plasmas," JHEP 0904 (2009) 137 [arXiv:0902.4696 [hep-th]].

[25] E. Witten, "Anti-de Sitter space, thermal phase transition, and confinement in gauge theories," Adv. Theor. Math. Phys. 2 (1998) 505 hep-th/9803131.

[26] V. Balasubramanian and P. Kraus, "A Stress tensor for Anti-de Sitter gravity," Commun. Math. Phys. 208 (1999) 413; S. de Haro, S. N. Solodukhin and K. Skenderis, "Holographic reconstruction of space-time and renormalization in the AdS / CFT correspondence," Commun. Math. Phys. 217 (2001) 595.

[27] H. Casini, M. Huerta and R. C. Myers, "Towards a derivation of holographic entanglement entropy," JHEP 1105 (2011) 036 [arXiv:1102.0440 [hep-th]].

[28] L. Bombelli, R. K. Koul, J. Lee and R. D. Sorkin, "A Quantum Source of Entropy for Black Holes," Phys. Rev. D 34 (1986) 373; M. Srednicki, "Entropy and area," Phys. Rev. Lett. 71 (1993) 666 [hep-th/9303048]. 
[29] J. Bhattacharya, M. Nozaki, T. Takayanagi and T. Ugajin, "Thermodynamical Property of Entanglement Entropy for Excited States," arXiv:1212.1164 [hep-th].

[30] J. D. Brown and M. Henneaux, "Central Charges in the Canonical Realization of Asymptotic Symmetries: An Example from Three-Dimensional Gravity," Commun. Math. Phys. 104 (1986) 207.

[31] C. Holzhey, F. Larsen and F. Wilczek, "Geometric and renormalized entropy in conformal field theory," Nucl. Phys. B 424, 443 (1994) arXiv:hep-th/9403108.

[32] P. Calabrese and J. Cardy, "Entanglement entropy and quantum field theory," J. Stat. Mech. 0406, P002 (2004) arXiv:hep-th/0405152.

[33] E. H. Lieb and M. B. Ruskai, "Proof of the strong subadditivity of quantum-mechanical entropy," J. Math. Phys. 14 (1973) 1938.

[34] H. Casini and M. Huerta, "A Finite entanglement entropy and the c-theorem," Phys. Lett. B 600 (2004) 142 [hep-th/0405111].

[35] M. Headrick and T. Takayanagi, "A Holographic proof of the strong subadditivity of entanglement entropy," Phys. Rev. D 76 (2007) 106013 [arXiv:0704.3719 [hep-th]].

[36] M. Fujita, T. Takayanagi and E. Tonni, "Aspects of AdS/BCFT," JHEP 1111 (2011) 043 arXiv:1108.5152 [hep-th]].

[37] T. Takayanagi, "Holographic Dual of BCFT," Phys. Rev. Lett. 107 (2011) 101602 arXiv:1105.5165 [hep-th]].

[38] B. Sundborg, "The Hagedorn transition, deconfinement and N=4 SYM theory," Nucl. Phys. B 573 (2000) 349 hep-th/9908001;

O. Aharony, J. Marsano, S. Minwalla, K. Papadodimas and M. Van Raamsdonk, "The Hagedorn - deconfinement phase transition in weakly coupled large N gauge theories," Adv. Theor. Math. Phys. 8 (2004) 603 hep-th/0310285].

[39] G. Vidal, "Entanglement renormalization," Phys. Rev. Lett. 99, 220405 (2007) , arXiv:cond-mat/0512165; "Entanglement renormalization: an introduction," arXiv:0912.1651; G. Evenbly and G. Vidal, "Quantum Criticality with the Multi-scale Entanglement Renormalization Ansatz," arXiv:1109.5334.

[40] B. Swingle, "Entanglement Renormalization and Holography," Phys.Rev.D 86 (2012)065007, arXiv:0905.1317 [cond-mat.str-el]; "Constructing holographic spacetimes using entanglement renormalization," arXiv:1209.3304 [hep-th].

[41] M. Nozaki, S. Ryu and T. Takayanagi, "Holographic Geometry of Entanglement Renormalization in Quantum Field Theories," arXiv:1208.3469 [hep-th].

[42] J. Haegeman, T. J. Osborne, H. Verschelde and F. Verstraete, "Entanglement renormalization for quantum fields," arXiv:1102.5524 [hep-th].

[43] J. Molina-Vilaplana and P. Sodano, "Holographic View on Quantum Correlations and Mutual Information between Disjoint Blocks of a Quantum Critical System," JHEP 1110 (2011) 011 [arXiv:1108.1277 [quant-ph]]; V. Balasubramanian, M. B. McDermott and M. Van Raamsdonk, "Momentum-space entanglement and renormalization in quantum field theory," arXiv:1108.3568 [hep-th]; H. Matsueda, "Scaling of entanglement entropy and hyperbolic geometry," arXiv:1112.5566 [cond-mat.stat-mech]; M. Ishihara, F. -L. Lin and B. Ning, "Refined Holographic Entanglement Entropy for the AdS Solitons and AdS black Holes," arXiv:1203.6153 [hep-th]; H. Matsueda, M. Ishihara and Y. Hashizume, "Tensor Network and Black Hole," arXiv:1208.0206 [hep-th]. 
[44] J. Ignacio Cirac and Frank Verstraete, "Renormalization and tensor product states in spin chains and lattices," J. Phys. A 42 504004, (2009), arXiv:0910.1130; G. Evenbly and G. Vidal,"Tensor network states and geometry," arXiv:1106.1082. 